\title{
AXL-TBK1 driven nuclear AKT3 promotes metastasis
}

Emily N. Arner ${ }^{1,2,{ }^{*}}$, Jill M. Westcott ${ }^{2}$, Stefan Hinz ${ }^{3,4}$, Crina Elena Tiron ${ }^{3,5}$, Magnus Blø ${ }^{4}$, Anja $\mathrm{Mai}^{4}$, Reetta Virtakoivu ${ }^{6}$, Natalie Phinney ${ }^{1,2}$, Silje Nord ${ }^{7}$, Kristina Y. Aguilera ${ }^{4}$, Ali Rizvi ${ }^{2}$, Jason E. Toombs ${ }^{2}$, Tanner Reese ${ }^{1}$, Vidal Fey ${ }^{6}$, David Micklem ${ }^{4}$, Gro Gausdal ${ }^{4}$, Johanna Ivaska ${ }^{6}$, James B. Lorens ${ }^{3, \#}$, Rolf A. Brekken ${ }^{1,2,8,9, \#}$

${ }^{1}$ Cancer Biology Graduate Program, ${ }^{2}$ Department of Surgery \& the Hamon Center for Therapeutic Oncology Research, ${ }^{8}$ Department of Pharmacology, University of Texas Southwestern Medical Center, Dallas, TX, 75390, USA

${ }^{3}$ Department of Biomedicine \& Centre for Cancer Biomarkers, University of Bergen, Norway

${ }^{4}$ BerGenBio ASA, Bergen, Norway

${ }^{5}$ Regional Institute of Oncology, lasi, Romania

${ }^{6}$ Turku Bioscience Center, University of Turku and Abo Akademi University, Department of Life Technologies, University of Turku, FIN-20520, Turku, Finland

${ }^{7}$ Department of Cancer Genetics, Institute for Cancer Research, Oslo University Hospital

${ }^{9}$ Lead Contact

${ }^{*}$ Current affliation: Division of Hematology/Oncology, Department of Medicine, Vanderbilt University Medical Center, Nashville, TN 37232 USA

Running title: Nuclear AKT3 drives metastasis

\section{\#Corresponding authors:}

Rolf A. Brekken, PhD

Hamon Center for Therapeutic Oncology Research

UT Southwestern Medical Center

6000 Harry Hines Blvd.

Dallas, TX 75390-8593 USA

rolf.brekken@utsouthwestern.edu
James B. Lorens, PhD

Department of Biomedicine

University of Bergen

Jonas Lies vei 91

N-5009 Bergen, Norway

jim.lorens@uib.no

Number of figures, tables: 8 figures, 6 supplemental, 2 supplemental table, 60,242 characters Declarations of interest: $R A B$ received research support from BerGenBio ASA for unrelated work; SH, AM, KYA, GG, DM, MB, JBL are or were employees of BerGenBio ASA; JBL and DM have ownership interest in BerGenBio ASA. The remaining authors do not have potential conflicts of interest. 
Arner et al.

\section{Abstract}

52 Epithelial-to-mesenchymal transition (EMT) contributes to tumor cell survival, immune evasion,

53 migration, invasion, and therapy resistance. Across human cancer, tumors that are high grade,

54 poorly differentiated, and have undergone EMT carry a worse prognosis with a higher likelihood

55 of metastasis. AXL, a receptor tyrosine kinase, drives EMT and is implicated in tumor

56 progression, metastasis, and therapy resistance in multiple cancer types including pancreatic

57 cancer and breast cancer. TANK-binding kinase 1 (TBK1) is central to AXL-driven EMT yet, the

58 mechanism of how TBK1 induces EMT remains unclear. Here, we report that AXL activation

59 stimulates TBK1 binding and phosphorylation of AKT3. TBK1 activation of AKT3 drives binding

60 and phosphorylation of slug/snail resulting in protection from proteasomal degradation and

61 translocation of the complex into the nucleus. We show that nuclear translocation of AKT3 is

62 required for AXL-driven EMT and metastasis. Congruently, nuclear AKT3 expression correlates

63 with worse outcome in aggressive breast cancer. To advance AKT3 as a therapeutic target, an

64 AKT3-isoform selective allosteric small molecule inhibitor, BGB214, was developed. BGB214

65 inhibits AKT3 nuclear translocation, EMT-TF stability, AKT3-mediated invasion of breast cancer

66 cells and reduces tumor initiation in vivo. Our results suggest that AKT3 nuclear activity is an

67 important feature of AXL-driven epithelial plasticity and that selective AKT3 inhibition represents

68 a novel therapeutic avenue for treating aggressive cancer.

\section{Significance}

71 Nuclear AKT3 activity is an important feature of AXL-TBK1 driven EMT and metastasis, thus

72 selective AKT3 targeting represents a novel approach to treat aggressive cancer.

73

74 Keywords: AKT3/AXL/EMT/invasion/TBK1 
Arner et al.

\section{Introduction}

Cancer metastasis, the leading cause of cancer mortality correlates with epithelial-tomesenchymal transition (EMT) $(1,2)$. Metastasis of epithelial tumors, such as pancreatic cancer (PDA), requires cancer cells to escape epithelial nests, invade surrounding stroma, intravasate into blood or lymphatic vessels, survive circulation, and extravasate at a secondary site, where successful cells form micrometastases and eventually macrometastases (3). The escape of tumor cells from tumor cell nests encapsulated by a basement membrane can be facilitated by tumor cell epithelial plasticity, which results in epithelial tumor cells losing contact with the basement membrane and nearby cells while adopting mesenchymal-like features that enhance cell migration and invasion. While epithelial plasticity alters morphology and cell-cell contact it also enables tumor cell survival under stressful environmental conditions, such as chemotherapy and radiation (4-7).

In carcinomas, the manifestation of an EMT program is associated with tumor grade. Highgrade cancer is characterized by a loss of normal tissue structure and architecture. High-grade tumors are often described as poorly differentiated, displaying tumor cells that have undergone full or partial EMT. In contrast, low-grade tumors that retain an epithelial phenotype are characterized as well-differentiated. Across human cancer, tumors that are high grade and poorly differentiated carry a worse prognosis with a high likelihood of metastasizing to distant organs (8). Understanding the molecular mechanisms underlying cellular plasticity and metastasis may reveal novel ways to target these programs for effective therapies.

Many signaling pathways can mediate tumor cell epithelial plasticity, including the receptor tyrosine kinase (RTK) AXL (9-11), elevated expression of which correlates with metastasis and resistance to therapy $(9,12)$. AXL is a member of the TAM (Tyro3, AXL, MerTK) family of RTKs (13) and is activated by its ligand, growth arrest-specific gene 6 (GAS6) to promote a variety of cellular processes, including epithelial plasticity, cell survival, proliferation and migration (9). We have previously shown that the serine threonine protein kinase TANK-binding kinase 1 (TBK1) 
Arner et al.

102

103

104

105

106

107

108

109

110

111

112

113

114

115

116

117

118

119

120

121

122

123

124

125

126

127

promotes EMT downstream of AXL in PDA, providing insight into a novel function for TBK1 (14). While the precise mechanism of how TBK1 drives EMT has yet to be determined, previous work found that TBK1 can directly activate AKT (15).

AKT is a key regulator of many cellular phenotypes associated with cancer, including cell survival, proliferation, and metastasis (16). Activation of AKT can drive EMT via the induction of EMT transcription factors (EMT-TFs) including snail and slug, which transcriptionally repress Ecadherin and induce vimentin, twist1, MMP-2, and MMP-9 that promote tumor cell invasion (7, 17, 18). There are three mammalian AKT isoforms (AKT1, AKT2, and AKT3). While each isoform is encoded by distinct genes, there is $\sim 80 \%$ amino acid sequence identity and each isoform appears to be activated by similar mechanisms $(19,20)$. Although the function of AKT in general in cancer cell survival and growth has been well characterized, the contribution of different AKT isoforms has not been investigated as intensely and is often under appreciated. Based on a phosphoproteomics screen, AKT isoforms have specific expression patterns and serve different functions in cell signaling and cancer (21). Although it is the least studied isoform, AKT3 has been implicated in various aspects of EMT, including tumor progression, DNA damage repair response, and drug resistance (22-25).

Here we report a novel mechanism in which nuclear AKT3 is vital to AXL-TBK1 driven EMT by stabilizing the EMT transcription factors slug and snail. Additionally, we report the first AKT isoform specific small molecule inhibitor, BGB214, which is an AKT3-isoform selective allosteric small molecule inhibitor. BGB214 inhibits EMT-TF stability, AKT3-mediated invasion, and tumor initiation in vivo. Lastly, we show that AKT expression drives metastasis in vivo and nuclear AKT3 expression correlates with aggressive cancer. Our findings suggest that nuclear AKT3 activity is an important feature of AXL-driven epithelial plasticity and that selective AKT3 targeting represents a novel therapeutic avenue for treating aggressive cancer. 
Arner et al.

128

129

130

131

132

133

134

135

136

137

138

139

140

141

142

143

144

145

146

147

148

149

150

151

152

\section{Results}

\section{AKT3 promotes EMT via TBK1}

$A X L$ activation promotes tumor cell migration and invasion (26). Consistent with this, $A X L$ mRNA expression correlates with EMT and stem cell-related gene expression in breast cancer cell lines and patient breast carcinoma biopsies, but not normal breast tissue (Supplemental Figure $1 \mathrm{~A}-\mathrm{C})$. Furthermore, IHC analysis of patient primary breast tumor biopsies revealed $\mathrm{AXL}$ protein expression correlates with expression of mesenchymal markers $\mathrm{N}$-cadherin and twist2 (Supplemental Figure 1D). Interestingly, analysis of publicly available GEO RNA sequencing data of breast cancer cell lines showed that while $A X L$ and $A K T 3$ correlate significantly, $A X L$ and $A K T 1$ or $A K T 2$ do not (Figure 1A). Similar results were found by analyzing the correlation of AKT isoforms and $A X L$ in human breast cancer using gene expression profiling interactive analysis (GEPIA) in invasive breast carcinoma (BRCA) from the TCGA database (Supplemental Figure 2A) (27). AKT1 and $A K T 2$ showed no correlation with $A X L$, whereas $A K T 3$ correlated significantly with $A X L$ expression in BRCA ( $p$-value $\left.=4.4 \times 10^{-110}, \mathrm{R}=0.61\right)$. In vitro, forced expression of slug in the epithelial breast line MCF10a (for cell line information see Supplemental Table 1) drives EMT and induces AXL and AKT3 expression, while AKT1 and AKT2 levels were not elevated (Supplemental Figure 2B). Additionally, when AXL was knocked down in these cells, AKT3 was no longer expressed (Supplemental Figure 2B), supporting the correlation between AXL and AKT3.

To investigate the function of AKT isoforms in EMT, MCF10a cells were treated for 4 days with TGF $\beta$, a potent EMT inducer, after which each AKT isoform was immunoprecipitated and probed for phosphorylation (S473). We found that TGF $\beta$-induced EMT results in phosphorylation of AKT3, but not AKT1 or AKT2, supporting that AKT3 is selectively associated with EMT (Supplemental Figure 2C). To further test the function of AKT3, CRISPR knockout of AKT3 was done in a primary pancreatic cancer cell line derived from 
Arner et al.

$153 \mathrm{Kras}^{L S L-G 12 D /+} \operatorname{Trp} 53^{f / f l} P d x 1^{\mathrm{Cre} /+}(K P F C)$, a genetically engineered mouse model (GEMM) of PDA.

154 In the absence of AKT3, mesenchymal markers zeb-1, vimentin, and slug were reduced, while

155 the epithelial marker E-Cadherin was increased in two different clones (AKT3 KO A and AKT3

$156 \mathrm{KO}$ B) compared to the Cas-9 empty vector (CAS9-EV) control, confirming the function of AKT3

157 in EMT (Figure 1B).

158 Given the correlation of AKT3 with AXL and EMT, we sought to determine if AKT3

159 contributes to AXL-mediated EMT. To mimic constitutively active AKT1 or 3, MCF10a cells were

160 transduced with retroviral vectors expressing myristoylated AKT1 (myrAKT1) or myristoylated

161 AKT3 (myrAKT3) and analyzed for changes associated with EMT (protein expression and

162 morphology, Supplemental Figure 2D, E). Transduction of myrAKT1 did not alter cellular

163 phenotype. However, myrAKT3 transduction resulted in robust changes in cell phenotype as

164 well as EMT protein changes. Expression of $\mathrm{AXL}$ and mesenchymal markers vimentin and N-

165 cadherin were elevated and the cells displayed a more invasive and mesenchymal-like

166 morphology in 2D and 3D (embedded in matrigel), suggesting constitutively active AKT3 can

167 drive EMT (Supplemental Figure 2D, E). To investigate if AKT3 is activated downstream of AXL,

168 PANC1 cells were treated with DMSO, GAS6, or GAS6 and a neutralizing monoclonal anti-AXL

169 antibody, tilvestamab. Probing for pAKT3 indicated that AKT3 can be activated in an AXL

170 specific manner (Supplemental Figure 2F).

171 Our prior studies established that TBK1 promotes EMT downstream of AXL in PDA (14).

172 Although the mechanism by which TBK1 drives EMT remains unclear, prior evidence shows

173 that TBK1 can directly activate $\operatorname{AKT}(15,16)$. Given our previous findings that AKT is activated

174 downstream of AXL in a TBK1-dependent manner (14) we hypothesized that TBK1 binds to and

175 activates AKT3 to drive EMT downstream of AXL. To test this, we treated MDA-MB-231 cells

176 with DMSO, GAS6, or GAS6 plus BGB324 (R428; bemcentinib), a small molecule AXL kinase

177 inhibitor $(12,28)$. Immunoprecipitation of TBK1 revealed that TBK1 binds to AKT3, and that AXL

178 stimulation results in the phosphorylation of TBK1 and AKT3 (Figure 1C). Furthermore, BGB324 
Arner et al.

179 inhibited GAS6-induced activation of TBK1 and AKT3. To investigate TBK1-AKT3 interaction

180 further, we used primary cell lines developed from GEMMs of pancreatic cancer, $T B K 1^{+/+} K I C$

$181\left(K r^{L S L-G 12 D /+} ; C d k n 2 a^{L o x / L o x} ; P t f 1 a^{C r e /}\right)$ or TBK1-mutant $\left(T B K 1^{\Delta / \Delta}\right) K I C$ mice (14), which are

182 deficient in TBK1 kinase activity. We found that AKT3 is phosphorylated in $T B K 1^{+/+} K I C$ cells but

183 not in $T B K 1^{\Delta \Delta} K I C$ cells (Figure 1D), supporting the hypothesis that TBK1 can activate AKT3. To

184 investigate if TBK1 can directly bind to and activate AKT3 we performed an in vitro kinase

185 activity assay with human recombinant TBK1 and AKT3 using cold ATP. Mass-spectrometry

186 analysis confirmed that TBK1 directly phosphorylates AKT3 at serine 472 (Supplemental Figure

$1872 \mathrm{2})$.

188 To investigate if AKT3 induces EMT downstream of TBK1, we transduced KIC TBK ${ }^{\Delta \Delta \Delta}$ cells 189 with myrAKT3 $\left(T B K 1^{\Delta \Delta} /\right.$ myrAKT3) and found that myrAKT3 rescues expression of

190 mesenchymal markers, vimentin and slug, and decreases the expression of E-cadherin (Figure

191 1E), demonstrating that myrAKT3 induces a mesenchymal-like phenotype in TBK1-mutant PDA

192 cells. To evaluate if the protein changes seen in Figure 1E result in a phenotypic change, we

193 cultured $T B K 1^{+/+}, T B K 1^{\Delta \Delta \Delta}$, and $T B K 1^{\Delta / \Delta} / m y r A K T 3 K I C$ cells in collagen/matrigel and found that

$194 \mathrm{TBK}^{+/+}$cells were invasive with elongated morphology while $T B K 1^{\mathrm{\Delta \Delta}}$ cells were epithelial and

195 less elongated (Figure 1F). Interestingly, $T B K 1^{\Delta / \Delta} /$ myrAKT3 cells reverted to a mesenchymal-like 196 morphology, similar to $\mathrm{TBK} 1^{+/+}$cells, suggesting constitutively active AKT3 is sufficient to drive

197 EMT, even in the absence of TBK1. These data support that AKT3 is downstream of TBK1 and

198 is required for TBK1 driven EMT.

$200 \quad$ AXL-TBK1 is required for AKT3 nuclear localization

201 It has been reported that while AKT1 and AKT2 are found in the cytoplasm and

202 mitochondria, respectively, AKT3 is often found in the nucleus (29). We observed clear nuclear

203 localization of AKT3 in MDA-MB-231 and MCF10A/slug cells (30) (Supplemental Figure 3A,B).

204 Interestingly, AXL silencing in MCF10a/slug cells reduced AKT3 nuclear localization, suggesting 
Arner et al.

205

206

207

208

209

210

211

212

213

214

215

216

217

218

219

220

221

222

223

224

225

226

227

228

229

230

that AXL mediates the nuclear localization of AKT3 (Supplemental Figure 3B). These data were validated further with cell fractionation experiments where AKT3 was primarily detected in nuclear fractions of MDA-MB-231 cells (Figure 2A) and HMLER cells transduced with myrAKT3 (Supplemental Figure 3C).

To determine if TBK1 contributes to the nuclear localization of AKT3, immunofluorescence of AKT3 in $T B K 1^{+/+}, T B K 1^{\Delta \Delta \Delta}$, and $T B K 1^{\Delta \Delta} / m y r A K T 3 K I C$ and MDA-MB-231 cells (Figure 2B, C) was performed. The percentage of cells with nuclear AKT3 was reduced $\sim 80 \%$ in the absence of functional TBK1 in KIC cells. This effect was partially rescued by myrAKT3, suggesting that AKT3 activation by TBK1 contributes to AKT3 nuclear localization. Furthermore, when MDAMB-231/GFP cells were treated with BGB324 to inactivate AXL, thereby preventing TBK1 activation, AKT3 did not translocate to the nucleus (Figure 2D). To investigate how AXL affects nuclear localization of AKT3, MDA-MB-231 (Supplemental Figure 3D) and PANC1 (Supplemental Figure 3E) cells were treated with serum free media (SFM), GAS6, or GAS6 + BGB324 for 12 hrs. Immunocytochemistry for AKT3 in MDA-MB-231 cells demonstrated that AKT3 was nuclear localized in $15.9 \%$ of cells treated with serum free media (SFM) while GAS6 treatment resulted in $47.1 \%$ of cells showing nuclear AKT3 (Supplemental Figure 3D). In contrast, AXL inhibition with BGB324, decreased nuclear AKT3 to only $2.9 \%$ of cells, supporting that $A X L$ stimulation induces the nuclear localization of AKT3. Similar effects were observed in PANC1 cells (Supplemental Figure 3E). To demonstrate that inhibition of AKT3 nuclear localization is not a general phenomenon associated with RTK inhibition, HMECs were treated with imatinib, an inhibitor of ABL/CKIT/PDGFR. BGB324 reduced nuclear AKT3 but the imatinib did not (Supplemental Figure 3F-G).

Proteins over 40 kDa must be actively transported through the nuclear membrane by importins, which recognize and bind nuclear location sequences (NLS) (31). We used a webbased NLS mapper (32) and identified a weak bipartite NLS in the AKT3 amino acid sequence (accession number: Q9Y243) located in a flexible linker region between the $\mathrm{PH}$-domain and 
Arner et al.

231 kinase domain (Supplemental Figure 4A). Based on these in silico findings we created two

232 AKT3 mutant overexpression constructs: AKT3-NLS1 and AKT3-NLS2. AKT3-NLS1 carries two

233 point-mutations (K141R and R142A) that alter the leucine rich NLS region to the sequence that

234 resembles the linker area in AKT2 (Supplemental Figure 4A). For AKT3-NLS2, a 10 amino acid

235 sequence flanking the NLS was replaced to mimic a longer part of the linker region as coded in

236 AKT2. Wildtype AKT3 and the mutants were retrovirally delivered and expressed in HMLER

237 cells (Supplemental Figure 4B). Immunocytochemical analyses showed clear subcellular

238 localization differences between control, AKT3, AKT3-NLS1 and AKT3-NLS2 transfected cells

239 (Supplemental Figure 4C). Wildtype AKT3 was predominantly (87\%) nuclear localized; however,

240 AKT3-NLS1 and AKT3-NLS2 mutants were largely restricted to the cytoplasm with 18\% and

$24129 \%$ nuclear localization, respectively. Immunoprecipitation of AKT3 in HMLER lysates and

242 probing with $\alpha$-importin showed that the AKT3-NLS1 mutant had impaired interaction with $\alpha-$

243 importin (Supplemental Figure 4D).

244 To further investigate the contribution of nuclear AKT3 to EMT, KPFC AKT3 KO cells were

245 transduced with wildtype AKT3 or AKT3-NLS1 and cultured in collagen/matrigel (Figure 2E).

246 Evaluation of invasion revealed that while AKT3 KO resulted in reduced invasive phenotype

247 compared to control, when wildtype AKT3 was rescued so was the invasive phenotype.

248 However, when AKT3-NLS was transduced into the AKT3 KO cells, invasion was no longer

249 rescued (Figure 2E), suggesting that nuclear AKT3 is necessary to drive EMT.

251 Snail and slug are AXL-TBK1 dependent substrates of AKT3

252 EMT is orchestrated by a limited number of transcription factors, considered to be the

253 ultimate inducers of EMT (EMT-TFs). These transcription factors include the zinc finger

254 transcription repressors snail $(S N A / 1)$ and slug $(S N A / 2)(2,33,34)$. Previously, we found that

255 the activation of TBK1 in AXL-driven metastasis drives the engagement of slug and snail (14).

256 We used GEPIA analysis to evaluate the correlation between the mRNA expression levels of 
Arner et al.

257

258

259

260

261

262

263

264

265

266

267

268

269

270

271

272

273

274

275

276

277

278

279

280

281

282

SNAI2 and the different $A K T$ isoforms in BRCA (Supplemental Figure 5A). Indeed, while AKT3 correlated with SNAI2 expression, AKT1 and AKT2 did not.

Given the presence of AKT3 in the nucleus, we sought to determine if AKT3 interacts with EMT-TFs. Immunoprecipitation of AKT3 in KIC PDA cells revealed that AKT3 associated with snail (Figure 3A). Furthermore, this complex remained intact in KIC PDA lines only when TBK1 was functional (Figure 3A), suggesting TBK1 is required for the interaction between AKT3 and snail. When PANC1 cells were treated with SFM or GAS6 (PANC1 cells produce GAS6, therefore there is a baseline level of GAS6-AXL signaling in cells treated with SFM) for 12 hrs, snail was found to be in the nucleus and cytoplasm of the cells (Figure 3B). However, when AXL was inhibited with BGB324, snail translocation to the nucleus was significantly reduced, suggesting that the AXL-TBK1-AKT3 pathway is involved in snail/slug nuclear translocation. Similar results were found with slug in MDA-MB-231 cells (Supplemental Figure 5B). This phenomenon was confirmed using imaging flow cytometry (Amnis Imagestream $®$ ), which clearly showed an $\sim 80 \%$ reduction of cells that display nuclear slug after AXL inhibition (Figure $3 C, D$ ).

\section{$\underline{\text { AXL activity stabilizes snail/slug via TBK1-AKT3 }}$}

Given the interaction between AKT3 and snail (Figure 3A) and the strong effect of AKT3 expression on snail and slug expression (Figure 1B), we hypothesized that slug/snail activity is dependent on AKT3 providing a stabilizing effect on slug/snail protein. To test this hypothesis, MCF10a/slug cells were transfected with siAKT3. Even though slug was overexpressed to drive EMT in these cells (30), when AKT3 was not present vimentin and AXL expression were substantially reduced (Figure 4A), supporting the hypothesis that AKT3 is required for slug/snail EMT-inducing activity.

To determine if AXL-AKT3 activity influences the protein stability of snail/slug, we treated PANC1 (Figure 4B) and MDA-MB-231 (Figure 4C) cells with cycloheximide (CHX), a protein synthesis inhibitor, +/- GAS6 over a time course of 6 hrs. Consistent with previous findings (35) 
Arner et al.

283

284

285

286

287

288

289

290

291

292

293

294

295

296

297

298

299

300

301

302

303

304

305

306

307

308

snail had a half-life of $1 \mathrm{hr}$ when treated with $\mathrm{CHX}$. Interestingly, when AXL was activated with GAS6, the half-life of snail was prolonged to $4 \mathrm{hrs}$, suggesting AXL activity stabilizes slug/snail protein. To determine AKT3 involvment, we repeated the experiment in cells transduced with shAKT3 and found that the addition of GAS6 no longer had a stabilizing effect on snail. AKT3 was similarly required for AXL-induced slug stabilty in MDA-MB-231 cells (Figure 4C). To determine whether snail protein is degraded by the proteasome or the lysosome, PANC1 cells were treated with a lysosome inhibitor (BafA1) or a proteasome inhibitor (MG-132) +/- GAS6 for 8 hrs (Supplemental Figure 5C). Although BafA1 had no effect on snail expression levels, when cells were treated with MG-132, there was a robust increase of snail protein, indicating snail is degraded via the proteasome.

The F-box E3 ubiquitin ligase FBWX7 has been implicated in the degradation of snail/slug in multiple cancers (36-38). Xiao and colleagues showed when FBXW7 was targeted with shRNA in two different lung cancer cell lines, the expression of snail increased markedly (36). This finding was recapitulated in ovarian cancer cells (37). To evaluate if AXL-AKT3 protects slug from FBXW7 and therefore degradation, we used Imaging flow cytometry (Amnis Imagestream ${ }^{\circledR}$ ) of MDA-MB-231 cells and scored co-expression of FBXW7 and slug (Figure 4D). Interestingly, when MDA-MB-231 cells were treated with GAS6, FBXW7 and slug were rarely overlapping, but when AXL was inhibited using BGB324, overlap of the two proteins dramatically increased, suggesting that perhaps AXL-TBK1-AKT3 protects slug from FBXW7 mediated degradation.

\section{Selective targeting of AKT3 with a novel allosteric small molecule inhibitor inhibits metastasis}

Several drugs targeting pan-AKT activity (e.g. GDC0068, AXD5363, MK-2206) are currently in various stages of clinical testing. However, many of these trials report toxicity such as hyperglycemia and hyperinsulinemia due to the essential functions of AKT1 and AKT2 in tissue homeostasis (39-42). An AKT3 selective inhibitor has the potential to overcome these issues. 
Arner et al.

309 The similarity between AKT1, 2 and 3 in the kinase domain precludes selective kinase inhibition.

310 However, an allosteric site located in a cleft between the $\mathrm{PH}$ domain and the kinase domain has

311 been used to identify AKT1, AKT2 and AKT1/2-selective inhibitors (43-45). Sequence alignment

312 around this allosteric site suggested that there are exploitable differences in this region (Figure

$3135 \mathrm{~A})$. A structural model produced by comparison of the crystal structures of AKT1 (45) and the

314 AKT2 kinase domain suggested that a single amino acid deletion in AKT2 and AKT3 compared

315 to AKT1 leads to a change in the path that the protein backbone follows, opening up a pocket at

316 the front of the allosteric binding site (Figure 5B). This pocket is small in the case of AKT2 due

317 to the protrusion of the large side chain of Arg269, but larger in AKT3 due to the presence of a

318 glycine at this site. A series of novel allosteric small molecule inhibitors of AKT3 were developed

319 (WO/2016/102672) with backbones that bind to the allosteric site via the right hand side of the

320 molecule with the group on the left making a bend to access the additional space, causing the

321 molecule to clash with AKT1 Lysine 268. One example of these is BGB214 (N-(5-(4-(1-

322 aminocyclobutyl)phenyl)-4-phenylpyridin-2-yl)-2-((1r,4r)-4-(N-

323 methylacetamido)cyclohexyl)acetamide), a potent and selective AKT3 inhibitor (Figure 5C). In

324 biochemical assays using purified tag-free enzymes, BGB214 had an IC 50 of 13 nM for AKT3

325 with approximately 1000 -fold selectivity against AKT1 and >35-fold selectivity against AKT2

326 (Figure 5D).

327 To evaluate the efficacy of BGB214 to prevent aggressive cancer traits such as migration

328 and 3D growth, MDA-MB-231 cells were plated in collagen/matrigel and treated with GAS6,

329 GAS6 + BGB214, or GAS6 + BGB324 for 48 hrs (Figure 5E, F). Invasion over 50 um was

330 determined and quantified revealing that inhibition of AXL or AKT3 substantially reduced cell

331 migration/invasion (Figure 5F). Similarly, in an organotypic 3D growth assay, BGB214 dose-

332 dependently prevented MDA-MB-231 growth (Supplemental Figure 6A), but did not significantly

333 affect cell growth in 2D proliferation assays (Supplemental Figure 6B, C). Interestingly, BGB214 
Arner et al.

334 inhibition in PANC1 cells resulted in decreased expression of total snail (Supplemental Figure $3356 \mathrm{D})$.

336 The specificity of BGB214 for PAKT3 was confirmed in a panel of cell lines in vitro and in

337 vivo. HMLER-AKT3 or HMLER-GFP cells were treated with increasing concentrations of

338 BGB214 (Supplemental Figure 6E). As HMLER-GFP cells have very low levels of AKT3

339 endogenously, pAKT levels were only reduced when AKT3 was overexpressed, indicating that

340 BGB214 selectively inhibits AKT3. In addition, MCF10-DCIS subcutaneous tumors treated with

$34125 \mathrm{mg} / \mathrm{kg}$ BGB214 for 2-6 days specifically resulted in decreased pAKT3 with little effect on

342 pAKT1 and pAKT2 (Supplemental Figure 6F).

343 To investigate the potential of BGB214 to prevent tumor initiation, HMLER cells transduced

344 with control vector or AKT3 were pre-treated in vitro with BGB214 for 24 hours and then injected

345 subcutaneously into NOD SCID mice at limiting dilutions $\left(1 \times 10^{5}-1 \times 10^{6}\right.$ cells $)$ and mice treated

346 with BGB214 for 14 days (Supplemental Figure 6G). AKT3 inhibition by BGB214 significantly

347 reduced the tumor initiation capacity of HMLER-AKT3 cells (Supplemental Figure 6G). The

348 same reduction in tumor initiating capacity was observed following injection of HMLER-AKT3

349 cells without in vitro treatment with BGB214 preceding injection (Supplemental Figure $6 \mathrm{H}$ ). We

350 conclude that inhibition of AKT3 with the allosteric inhibitor BGB214 prevents AKT3 mediated

351 tumorigenic features such as invasion, 3D growth, EMT transcription factor stability and tumor

352 initiation.

354 AKT3 expression is associated with poorly differentiated tumors and increased metastasis

355 To assess the biologic consequence of AKT3, control KPFC cells (CAS9-EV), AKT3 KO

356 KPFC cells (AKT3 KO), or AKT3 KO KPFC cells transduced with AKT3 (Rescue) were injected

357 orthotopically into the pancreas of C57BL/6J mice (Figure 6). Primary tumor and metastastic

358 burden was evaluated 19 days post injection. Although tumor weight did not differ significantly

359 between the three groups (Figure 6B), gross metastatic burden was reduced in tumors lacking 
Arner et al.

AKT3 (Figure 6A). H\&E analysis as well as CK19 (a PDA tumor cell marker, (46) IHC confirmed significantly reduced metastasis to livers of AKT3 KO tumor-bearing mice (Figure 6C-D).

Consistent with this observation, tumors lacking AKT3 were more well-differentiated and expressed higher levels of E-Cadherin and lower levels of vimentin (Figure 6E). The expression of E-Cadherin and vimentin in vivo was consistent with the expression of these proteins in vitro (Figure 6F). Importantly, we observed that the expression level of AKT3 also correlated with the number of gross metastases (Figure 6A, F).

\section{Nuclear AKT3 is associated with aggressive cancer and worse survival in patients}

We next sought to evaluate the importance of nuclear AKT3 in cancer patients. To assess the location of AKT3 in pancreatic tumors from patients, IHC for AKT3 and AXL (Figure 7A) demonstrated that $\mathrm{AXL}^{+}$tumors displayed single cells outside epithelial ducts that expressed nuclear AKT3. However, in AXL- tumors, AKT3 was cytoplasmic, supporting our findings that $A X L$ is associated with nuclear localization of AKT3 and this localization results in a less differentiated (more mesenchymal-like) tumor cell phenotype (Figure 7A).

To assess the effect of AKT3 expression in breast epithelial cells, we retrovirally overexpressed AKT3 in MCF10A cells and compared the mRNA expression pattern via RNA sequencing with MCF10A cells transduced with GFP control vector. We found 46 differentially expressed (DE) genes (FC $\geq 2, F D R<0.05)$ (Supplemental Table 2). The DE genes and their directionality were used to calculate an "AKT3 score" which was then mapped against probes in the Metabric database, which is composed of gene expression patterns from 1980 breast cancer patients. The patients were divided into two groups depending upon if the AKT3 score was above or below the mean. Plotting the AKT3 score against patient survival indicates that a high AKT3 score correlates with a significantly worse overall outcome (KM, $p=8 e-9)$ (Figure 7B). Further, we found a significantly different distribution between breast cancer subtypes (based on PAM50 intrinsic subtypes) and AKT3 scores ( $p=4.3 \times 10^{-155}$, Kruskal-Wallis test) (Figure 7C). ER 
Arner et al.

negative tumors are in general enriched for the AKT3 score, particularly basal-like tumors

(Figure 7C). That high levels of AKT3 associated gene expression correlates with more aggressive forms of breast cancer, worse overall outcome, and a higher hazard ratio is consistent with previous reports of AKT3 high copy number alterations in TNBC patients (47, 48) and reports that AKT3 expression is associated with higher grade breast cancer tumors

391 (49). To validate our previous findings, we sought to determine if nuclear AKT3 was associated

392 with worse overall survival. To evaluate this, we performed IHC for AKT3 in clinical breast

393 cancer samples (Figure 7D, E). Grouping patients based on AKT3 subcellular localization

394 revealed that nuclear AKT3 predicted a worse overall outcome ( $n=53$ patients, $p=0.0013$ Log-

395 rank test) in this cohort of patients. Together, these results suggest that nuclear AKT3 may be a

396 therapeutic target that avoids toxicity associated with pan-AKT inhibition and a biomarker for 397 worse overall survival and aggressive cancers.

Discussion

We report that $A X L$ activation by its ligand GAS6 leads to the stimulation of TBK1 and

401 subsequent selective activation of the AKT isoform, AKT3. Activation of AKT3 drives the binding 402 of AKT3 to its substrate slug/snail, and translocation into the nucleus. The binding of AKT3 to 403 slug/snail also protects the EMT-TFs from proteasomal degradation, potentially by preventing 404 ubiquitinylation by FBWX7 (Figure 8). These results highlight the function of AKT3 in EMT and 405 its potential value as a therapeutic target, inhibition of which could enhance sensitivity to 406 standard therapy.

407 AKT activation is linked to fundamental signaling pathways underlying cancer development 408 and progression. Many investigations have focused on the function of AKT1, AKT2, or panAKT, 409 but have largely ignored AKT3. This may be because AKT3 is the least expressed of the three 410 isoforms (50) and prior results on the function of AKT3 in tumorigenesis are inconsistent (22,

411 51-55). Regardless, a few reports have suggested that AKT3 contributes to cancer progression, 
Arner et al.

412 including breast cancer (56-59). We predict that the inconsistency of AKT3 studies may be due

413 to differential genetic contexts of the studies as well as to the fact that AKT3 can be expressed

414 as two alternatively spliced variants, one which lacks S472 (60). In the study by Suyama et al.,

415 overexpression of the AKT3 variant lacking S472 was associated with improved overall survival

416 and reduced lung metastasis in preclinical models of breast cancer, whereas when AKT3 had

417 the S472 phosphorylation site they saw increased tumorigenesis (60). This is consistent with

418 our findings that phosphorylation on serine 472 via TBK1 is needed for AKT3 nuclear

419 localization to promote EMT and metastasis.

420 Our previous studies have shown an increase in other EMT-TFs, such as ZEB1,

421 downstream of AXL-TBK1 (14). Further studies are needed to evaluate if the AXL-TBK1-AKT3

422 signaling cascade only influences protein expression of snail/slug or multiple EMT-TFs.

423 Additionally, in our study, we only interrogated this pathway in the context of $A X L$ and $A X L$

424 stimulation. It is possible that this mechanism may only be relevant in cell lines that contain high

425 levels of $A X L$, which is supported by our finding that $A X L$ is expressed in human PDAC tumors

426 that display the nuclear localization of AKT3. Further studies are needed to understand if other

427 RTKs can activate TBK1-AKT3 to stabilize snail/slug.

428 In our study we find that the binding of AKT3 to slug/snail protects the EMT-TFs from

429 proteasomal degradation, although more studies are needed to determine if FBXW7 is required

430 for the degradation of slug/snail. It is possible that AKT3 does not directly stabilize EMT-TFs,

431 but perhaps other proteins such as deubiquitinating enzymes (DUBs) promote the stability of

432 these EMT-TFs in an AXL-TBK1 dependent manner. For example, the DUB USP10 has been

433 shown to promote the stability of slug and snail in breast, ovarian, and lung cancer cell lines

434 (61). Other potential candidate proteins that might be involved in the degradation of snail are the

435 F-box ligases, of which FBX15 and FBX011 have been shown to ubiquitinate and support the

436 degradation of Snail (62). Another protein that has been implicated in regulating the expression

437 of slug and $\mathrm{AXL}$ is the transcription factor $\Delta \mathrm{Np63a}$, which has been shown to drive the migration 
Arner et al.

438 of basal breast cancer cells in part through elevation of the expression of AXL and slug, as well

439 as miR-205 to silence ZEB1/2 (63). $\Delta$ Np63a drives breast cancer invasion by selectively

440 engaging certain proponents of the EMT program while still promoting the retention of epithelial

441 characteristics to drive collective migration. Further studies are needed to evaluate the exact

442 pathway by which slug/snail is degraded in an AXL-TBK1-AKT3 dependent manner.

$443 \quad$ These studies do not rule out that AKT3 affects other cell types such as macrophages; this

444 is relevant given the function of TBK1 in innate immune signaling and the STING pathway. In

445 fact, it has been reported that 7-DHC, a cholesterol precursor, regulates type I interferon

446 production via AKT3 activation, where AKT3 directly binds and phosphorylates IRF3 on S385

447 (64). Additionally, AKT3 (pS473) in macrophages has been shown to promote migration,

448 proliferation, wound healing, and collagen organization (65). Interestingly, a recent study

449 showed that AKT3 phosphorylated RNA processing proteins that regulate the alternative

450 splicing of fibroblast growth factor receptors (FGFR), consistent with an importance of nuclear

451 targeting of AKT3 in EMT maintenance (21).

452 In summary, our data support that nuclear AKT3 has utility as a potential biomarker for

453 aggressive cancers that express $A X L$, and that AKT3 is a specific mediator of EMT signaling

454 downstream of $A X L$. Additionally, as there are ongoing clinical trials targeting $A X L$ in multiple

455 cancer types, analyses of these tumors for AXL expression and AKT3 localization after

456 treatment may provide clinicians with a much-needed read-out for treatment efficacy. Lastly, we

457 propose that selective inhibition of AKT3 may represent a novel therapeutic avenue for treating

458 aggressive and recurrent cancer that avoids toxicity associated with pan-AKT inhibition. 
Arner et al.

\section{Materials and Methods}

\section{Reagents}

The following antibodies were used for immunoblotting (IB) at 1:1,000 unless otherwise stated: Anti-AXL (8661S, Cell Signaling, IHC 1:500), anti-phospho AXL y702 (5724, Cell Signaling), anti-phosphoserine (AB1603, Millipore), anti-TBK1 (3013S, Cell signaling), anti-TBK1 (ab40676, Abcam, IF 1:250), anti-TBK1 (NB100-56705AF647, Novus, FC 1:100), anti-pTBK1 s172 (5483S, Cell Signaling), anti-SNAIL (3879, Cell Signaling), anti-SLUG (9585, Cell Signaling), anti-SLUG-AF488 (NBP2-74235AF488, Novus, FC 1:100), anti-E-cadherin (clone 24E10, 3195S, Cell Signaling; IB 1:1000; IHC 1:400), anti-N-cadherin (14215S, Cell Signaling), anti-BCatenin (8480, Cell Signaling), mouse anti-human Twist (Twist2C1a, Abcam, IHC), a-actin (A2066, Sigma, 1:2000), anti-a-tubulin (T6199, Sigma), anti-Vimentin (5741, Cell Signaling; IB 1:1000; IHC 1:400), anti-CK19 (ab52625, abcam; IHC 1:1000), anti-AKT1 (2967, Cell Signaling), anti-AKT2 (3063, Cell Signaling), anti-AKT3 (1586912, Millipore, IHC), anti-AKT3 (14982, Cell Signaling, IP, IB, and IF 1:250), anti-AKT3 (HPA026441, Sigma, IHC 1:200), antiAKT3-PE (NBP2-71528PE, Novus, IF 1:250, FC 1:100), anti-pAKT (Ser473) (2971, Cell Signaling), anti-Lamin A/C IgG2b (Santa Cruz, sc-7292), anti-AKT (Cell signaling Technology, 9272), anti-Importin a (I1784, Sigma), anti-GAPDH (2118, Cell Signaling), anti-Phalloidin-AF546 (A22283, Invitrogen, IF 1:500), Hoechst 33342 (IF, 1:2000) and anti-FBXW7-PECY7 (NBP250403PECY7, Novus, FC 1:100). The following reagents were purchased from Sigma: Cycloheximide (01810-1g), BafA1 (B1793-2UG), MG-132 (474787-10MG). The CRU5-IRESGFP retroviral vectors for expression of hSNAIL, hSLUG, myrAKT1, myrAKT3, AKT3, shLuc, and shAXL (RFP) were prepared as described (11). CRU5-IRES-GFP retroviral vectors for expression of AKT3-NLS and AKT3-NLS were generated by site-directed mutagenesis (Quik change \#2200519). CRU5-IRES-GFP Luciferase AKT3-Luciferase and AKT3-NLS1-Luciferase were generated by cloning. All vectors were confirmed by DNA sequencing. Lentiviral shRNA 
Arner et al.

485

486

487

488

489

490

491

492

493

494

495

496

497

498

499

500

501

502

503

504

505

506

507

508

509

constructs against human AKT1, AKT2, AKT3, and TBK1 were purchased from Dharmacon (TBK1, RHS3979-201735457, clone ID: TRCN0000003184)(AKT1, RHS3979-201768650, clone ID: TRCN0000039797)(AKT2, RHS3979-201732837, TRC00000005630)(AKT3, RHS3979201733886, TRCN0000001612). Retroviral production and infections were conducted using Phoenix A retroviral packaging cells as described (66). Lentiviral production and infection were conducing using HEK293T cells as previously described (14). Human Gas6 from conditioned media was prepared as previously described (9). AKTVIII (Sigma), Imatinib (LC laboratories I5508) and BGB324/R428, BGB214, were prepared in DMSO. Cell culture, retroviral transductions, siRNA transfection HMEC strains (4th passage) were established and maintained as described (67) in M87A medium with oxytocin and cholera toxin (68). PANC1, MDA-MB-231, 4T1, and MCF10A (American Type Culture Collection, Rockville, MD) cells were cultured as described (11). HMLE and HMLER cells (a gift from Dr. R. Weinberg) were maintained as per Mani et al (69). TBK1 WT and deficient KIC murine cell lines were established and maintained as previously described (14). siRNA transfections were conducted as previously described (70).

\section{CRISPR Knockout}

Oligos of the gRNAs were annealed with T4 polynucleotide kinase (New England Biolabs) by PCR. Annealed oligos were then ligated to PX458 vector with FastDigest BbsI (FD1014, Thermo Fisher Scientific) and T7 DNA Ligase (M0318, New England Biolabs) by PCR. Mixture from the reaction was then transformed into the NEB 5- $\alpha$ Competent E. coli (High Efficiency). DNA was extracted from expanded colonies and sent to UTSW sequencing core for sequencing. Plasmids with correct gRNA sequences or empty vector control were transfected into KPFC cells with Lipofectamine 2000 (11668027, Thermo Fisher Scientific). Positive cells expressing GFP were sorted as single clones and expanded. Each expanded clone was subjected to validation through PCR and western blot analysis. 
Arner et al.

510

511

512

513

514

515

516

517

518

519

520

521 Immunoblotting and Flow Cytometry

522 Western blot analysis and flow cytometry analysis of cell lines were conducted as previously

523 described (11). MCF10A cells were treated with TGF $\beta(10 \mathrm{ng} / \mathrm{ml})$ for 4 days and then lysed

524 using NP40 Cell Lysis Buffer (40 mM HepesNAOH, 75 mM NaCl, 2 mM EDTA, 1\% NP40,

525 phosphatase inhibitor cocktail tablet, protease inhibitor cocktail tablet (Roche)). For

526 immunoprecipitation, antibodies against separate AKT isoforms (1, 2 and 3) and control lgG

527 antibodies $(1 \mu \mathrm{g} / \mathrm{lys}$ ate $)$ were added to lysates and incubated overnight at $4^{\circ} \mathrm{C}$. Next day the pre-

528 blocked protein-A/G beads (GE Healthcare) in lysis buffer were added and allowed to bind at

$5294^{\circ} \mathrm{C}$ for $1 \mathrm{hr}$. Beads were then washed 3 times $(20 \mathrm{mM}$ Tris- $\mathrm{HCl}(\mathrm{pH} 7,5), 150 \mathrm{mM} \mathrm{NaCl}, 1 \%$

530 NP40) and protein eluted by boiling in SDS-PAGE loading buffer. Running of SDS/PAGE gel

531 and immunoblotting were carried out according to standard procedures. Membranes were

532 probed using anti-pAKT (Ser473) and Pan-AKT antibodies. Nuclear extraction of MDA-MB-231

533 cells was done according to manufacturer's instructions (Universal Magnetic Co-IP Kit, Active

534 Motif, 54002). Imaging flow cytometry analysis was conducted on an Amnis Imagestream Mk

535 (>100,000 events) using the Imagestream software (Tree Star, Inc., Ashland, OR, USA) in the 
Arner et al.

536 Flow Cytometry Core at UT Southwestern. All Western and flow cytometry results shown were

537 performed in at least three independent experiments.

\section{Biochemical assays for AKT activity}

540 An Akt activation assay was used in which PDK1 was used to phosphorylate inactive Akt

541 enzymes, which then phosphorylated a GSK3a-derived LANCE Ultra Ulight-labelled crosstide

542 substrate (Perken Elmer, TRF0106-M). Addition of a Europium-labelled antibody specific to the

543 phosphopeptide (LANCE Ultra Europium-anti-phospho-Crosstide (anti-GSK-3a Ser21, Perkin

544 Elmer, TRF0202-M) allows proximity-dependent energy transfer from the Europium donor to the

545 Ultra Ulight ${ }^{\mathrm{TM}}$ acceptor. Briefly, $5 \mu \mathrm{L}$ enzyme in 1X AB (50 mM HEPES pH 7.5, 1 mM EGTA, 10

$546 \mathrm{mM} \mathrm{MgCl}_{2}, 0.01 \%$ Tween, $2 \mathrm{mM} \mathrm{DTT}$ ) was incubated with $2.5 \mu \mathrm{L}$ test compound. To start the

547 reaction $2.5 \mu \mathrm{L}$ reaction mix was added which consisted of PDK1, lipid preparations, crosstide

548 and ATP in $1 X$ AB. Final assay concentrations were: $1 \%$ DMSO, 5 nM Akt1/5-15 nM Akt2/3-5

$549 \mathrm{nM}$ Akt3 as appropriate, $5 \mathrm{nM}$ PDK1, $5.5 \mu \mathrm{M}$ DOPS, $5.5 \mu \mathrm{M}$ DOPC, $0.55 \mu \mathrm{M}$ Ptdlns $(3,4,5) \mathrm{P}_{3}$,

$550100 \mu \mathrm{M}$ ATP, $100 \mathrm{nM}$ crosstide. After $30 \mathrm{~min}$, the reaction was stopped using $5 \mu \mathrm{L} 40 \mu \mathrm{M}$ EDTA

551 in 1X LANCE Detection buffer (Perkin Elmer, CR97-100) for $5 \mathrm{~min}$. For detection, $5 \mu \mathrm{L} 8 \mathrm{nM}$

552 Europium-anti-phospho-Crosstide antibody in 1X Detection buffer was added to each well and

553 incubated for $1 \mathrm{~h}$. Plates were read with an EnVision® Multilabel Plate Reader, excitation at 320

$554 \mathrm{~nm}$ and emission at $665 \mathrm{~nm}$ and $615 \mathrm{~nm}$. Results were converted to percent inhibition of

555 phosphorylation by normalizing to positive and negative controls, and compound $\mathrm{IC}_{50}$ was

556 determined using a 3-parameter equation (Prism, GraphPad).

558 3D culture experiments

559 Growth factor reduced Matrigel (Corning, 10-12 mg/mL stock concentration, catalog no.

560354230 ) and bovine (Corning, catalog no. 354231) or rat tail (Corning, catalog no. 354236)

561 Collagen I were used for organotypic culture experiments. Vertical invasion assays and 
Arner et al.

562 experiments in three-dimensional (3D) culture were performed and quantified as described

563 previously (71) using a Matrigel/Collagen I matrix (3-5 mg/mL Matrigel and $1.8-2.1 \mathrm{mg} / \mathrm{mL}$

564 Collagen I). A 120- 4 m span on the z-axis is shown for the vertical invasion assays.

565

566 Mammosphere and tumorsphere formation assay

567 Mammosphere cultures were performed as previously described (72). Single cells were plated

568 in ultra-low attachment plates (Corning, Acton, MA, USA) at a density of 20,000 viable cells $/ \mathrm{ml}$.

569 Total mammospheres per well were quantified using ImageJ.

570

571 Gene Expression Analysis and RNA sequencing

572 The expression analysis of the breast cancer cell lines and human samples (cancer, normal)

573 was performed from published and GEO-submitted Affymetrix data as described (Kilpinen et al.,

574 2008). Global gene expression analysis of HMEC lineage was performed on FACS sorted

575 (FACSVantageSE) pre-stasis HMEC (4th passage) cells. Total RNA from FACS-enriched

576 primary culture cells were isolated with TRIzol (Invitrogen) and RNeasy Mini column (Qiagen)

577 and evaluated using Bioanalyzer (Agilent Technologies). Gene expression levels were

578 measured using the Illumina HumanHT-12 v4 Expression BeadChip whole-genome expression

579 array. The Illumina Bead Array data were quality controlled in Genome Studio and both probe

580 level and gene level data were imported into JExpress Pro (http://jexpress.bioinfo.no) for

581 analysis. After quantile normalization both datasets were log2 transformed. Correspondence

582 Analysis (Fellenberg et al., 2001) was performed on the datasets, together with Hierarchical

583 Clustering of the samples using a Pearson correlation measure on a per gene mean centered

584 version of the data. Differentially expressed genes between $\mathrm{AXL}^{+}$and $\mathrm{AXL}^{-}$groups were

585 identified using the Rank Product method on both datasets (Breitling et al., 2004). The resulting

586 lists of differentially expressed genes with a false discovery rate value $q=10 \%$ from these two

587 analysis was considered differentially expressed between the two groups. Cells were plated on 
Arner et al.

$58810 \mathrm{~cm}$ dishes until cell densities of $70 \%$ were achieved. Total RNA was extracted from cells

589 using QIAGEN RNeasy Mini kit and stored at $-80^{\circ} \mathrm{C} .1 \mu \mathrm{g}$ total RNA per sample were subjected

590 to library generation using the TruSeq stranded total RNA sample preparation kit, according to

591 the manufacturer's protocol (Illumina). The libraries were pooled and sequenced on a NextSeq

592500 instrument (high output flowcell) at 1x75 bp single end reads (Illumina). Raw RNAseq reads

593 were aligned against to the human genome release GRCh38/hg38 using HISAT2 (73) and

594 exons were counted using RSubread.featureCounts (74). Libraries were filtered to remove gene

595 counts of less than 1 CPM across all libraries and normalized. Differentially expressed genes

596 between GFP control group and AKT3 overexpressing MCF10A cells were calculated using

597 edgeR $(75,76)$. Genes were considered differentially expressed with a fold change $>2$ and

$598 \quad p<0.05$.

600 AKT3 score and Metabric dataset

601 To assess the influence of AKT3 signaling and its downstream targets on survival of breast

602 cancer patients, genes that were found to be differentially expressed after AKT3 overexpression

603 in MCF10A cells were used to generate an AKT3 score. The score essentially represented the

604 sum of expression of 42 differentially expressed genes, adjusted for expected directionality.

605 Initially, we examined 46 different genes, but only 42 of them were represented with probes on

606 the expression array. For genes represented by multiple probes (the 42 genes mapped to 71

607 different probes), mean signal intensity was used. The influence on breast cancer specific

608 survival and the putative difference between molecular subtypes was investigated in the

609 Metabric cohort, composed of 1980 breast cancer patients enrolled at five different hospitals in

610 the UK and Canada (77). Gene expression was assessed using the Illumina HT-12 v3

611 microarray and normalized data was downloaded from the European Genome-phenome

612 Archive (EGA) data portal. Missing values were imputed using the impute.knn function as

613 implemented in the R library 'impute' with default settings (Hastie T, c R, Narasimhan B and 
Arner et al.

614 Chu G (2016). Impute: Imputation for microarray data. R package). The data was batch

615 adjusted for hospital effect using the pamr.batchadjust function in the 'pamr' library with default

616 settings (T. Hastie, R. Tibshirani, Balasubramanian Narasimhan and Gil Chu (2014). Pam:

617 prediction analysis for microarrays). Association between the score and molecular subtypes (77,

618 78) was tested using Kruskal-Wallis rank test, and correlations were estimated with Spearman's

619 rank correlation. Survival analyses were performed using Cox proportional hazards regression

620 model as implemented in the R library 'rms' (Frank E Harrell Jr (2016). rms: Regression

621 Modeling Strategies). Survival plots were generated using the survplot function, as implemented

622 in the rms library. All analyses were performed using $\mathrm{R}$ version 3.3.1.

\section{Confocal Microscopy}

625 Cells were plated on coverslips (79.5, Marienfeld-Superior) overnight under low serum (1\%)

626 conditions. Cells were fixed with $4 \%$ formaldehyde diluted in warm PBS for 15 min, washed 3

627 times, blocked, and permeabilized with $5 \%$ goat serum, $0.3 \%$ Triton X100 in PBS for $1 \mathrm{~h}$. Cells

628 were incubated with the appropriate primary antibody overnight followed by 3 wash steps with

629 PBS and secondary antibody incubation for $2 \mathrm{~h}$ in $5 \%$ BSA in PBS. After 3 wash steps with PBS,

630 coverslips were mounted on slides with Prolong Diamond Antifade Reagent (Thermo Fisher).

631 The images were acquired using Leica SP5, Leica SP8, Zeiss LSM780, or Zeiss LSM880

632 inverted microscopes.

634 Immunohistochemistry

635 Paraffin-embedded Human PDAC samples were provided by the Tissue Management Shared

636 Resource within the Simmons Comprehensive Cancer Center at UT Southwestern. Both AXL

637 and AKT3 antibodies were optimized and stained using a Leica Autostainer. Paraffin-embedded

638 normal human breast tissue sections ( $n=20$; generously provided by Dr. A.Borowsky) were

639 prepared for immunofluorescence and stained with as previously described (Garbe et al., 2012). 
Arner et al.

640 For $\mathrm{N}$-cadherin analysis, antigen retrieval was performed by boiling for 20 min at in Tris EDTA

641 buffer, ph 9 in a microwave oven. A Dako Autostainer was used for staining. The slides were

642 incubated 60 minutes at room temperature with a monoclonal antibody against $\mathrm{N}$-cadherin

643 (M3613), dilution 1:25 (Dako). Immunoperoxidase staining was carried out using the Dako

644 Envision Kit with diaminobenzidin tetrachloride peroxidase. For analysis of Twist-2, antigen

645 retrieval was performed by boiling in TRS buffer ( $\mathrm{pH}$ 6.0) (Dako) for 25 minutes, and incubated

646 for $1 \mathrm{hr}$ in room temperature with the rabbit polyclonal antibody Twist-2 diluted 1:500, and

647 stained with HRP EnVision rabbit (Dako) for 30 minutes in RT. The peroxidase was localized by

648 the diaminobenzidine tetrachloride peroxidase reaction and counterstained with Mayer`s

649 hematoxylin. For Axl analysis, the sections were boiled in TRS buffer (pH 6.0) (Dako) in 20

650 minutes, followed by incubation overnight at room temperature with goat IgG antibody Axl,

651 dilution 1:50 (R\&D AF854) and stained with EV rabbit for 30 minutes. The peroxidase was

652 localized by the diaminobenzidine tetrachloride peroxidase reaction and counterstained with

653 Mayer's hematoxylin. The human breast cancer tumor sections were obtained from the IRO

654 database and assayed for quality control by a pathologist. IHC staining was carried out using

655 DAKO, EnVision ${ }^{\mathrm{TM}}$ FLEX kit with DAB before counterstaining with hematoxylin (DAKO,

656 EnVision ${ }^{\mathrm{TM}}$ FLEX Hematoxylin K8008). Stained samples were acquired using with Zeiss Axio

657 Observer Z1 microscope and analyzed with TissueGnostics software for acquisition and

658 analysis. Representative regions were analyzed from each sample slide and mean intensity of

659 DAB-AKT3 staining from nuclei and cytoplasm was used to separate nuclear AKT3 cases from

660 cytoplasmic AKT3 cases.

\section{Animal studies}

663 Sygeneic pancreatic cancer model: 
Arner et al.

KPFC (CAS9-EV, AKT3 KO, Rescue) cells were injected orthotopically (2.5 × $10^{5}$ cells) in 6- to 8-week-old C57BL/6 mice. 19 days after tumor cell injection mice were sacrificed and organs

668 were harvested for analysis. All animals were housed in a pathogen-free facility with 24-h

669 access to food and water. Animal experiments in this study were approved by and performed in

670 accordance with the institutional animal care and use committee at the UTSW Medical Center at

671 Dallas. Before implantation, cells were confirmed to be pathogen free.

672

673 Tumor cell titration studies:

674 Xenograft tumor-initiation studies were conducted as described by (80). HMLER cells (GFP, 675 myrAKT1, myrAKT3 or AKT3) were suspended in DMEM/Matrigel (1:1) in $50 \mu \mathrm{L}$ ) and injected 676 subcutaneously into 3-6 weeks old NOD-SCID mice. Tumor incidence was monitored with hand 677 held caliper for up to 60 days after injection; tumor threshold was set at $20 \mathrm{~mm} 3$ (AKT3) or 25 $678 \mathrm{~mm} 3$ (myrAKT3). Animals were treated with BGB214 dissolved in 0.5\% HPMC/0.1\% Tween 80

679 (Vehicle) as indicated in figure legends starting at the day of cell injection. For some studies, 680 cells were treated for $24 \mathrm{hrs}$ with $0.54 \mu \mathrm{M}$ BGB214 prior to implantation. Tumor cell titration 681 animal experiments were approved by The Norwegian Animal Research Authority 682 andperformed in accordance with The European Convention for the Protection of Vertebrates 683 Used for Scientific Purposes.

\section{In vitro kinase assay}

$6861.5 \mu \mathrm{g}$ of recombinant GST-AKT3 (BML-SE369-0005, Enzo Life Sciences), $0.1 \mu \mathrm{g}$ of

687 Recombinant Active TBK1 (T02-10G-05, SignalChem), and $1 \mu \mathrm{L} 10 \mathrm{mM}$ ATP were combined in 688 kinase reaction buffer $(20 \mathrm{mmol} / \mathrm{L}$ Tris-HCl pH 7.4, 500 mmol/L $\beta$-glycerol phosphate, 12 $689 \mathrm{mmol} / \mathrm{L}$ magnesium acetate) up to a total of $30 \mu \mathrm{L}$. Kinase reaction was carried out at $30^{\circ} \mathrm{C}, 500$ $690 \mathrm{rpm}$ for $1 \mathrm{hr}$. After reaction, AKT3 protein was resolved by SDS-PAGE and stained by 
Arner et al.

691 Coomassie Brilliant Blue. Bands were cut out for MS analysis to identify phosphorylation site by

692 the UT Southwestern Proteomics core.

\section{Statistical analysis}

695 GraphPad Prism 5.0 for PC, and MatLAB were used for statistical analysis using tests stated in

696 the Figure Legends. Comparisons of histological SI groups were performed by Pearson $\mathrm{X}^{2}$ test

697 using cut-off values for staining index (SI) categories based on median values. Grouped

698 analyses were performed with Bonferroni's test for multiple comparisons. Significance was

699 established when $p<0.05$.

\section{Author contributions}

702 ENA, JBL, and RAB conceived and designed the study. ENA, JMW, SH, CET, MB, AM, AV, NP,

$703 \mathrm{SN}, \mathrm{AR}, \mathrm{JET}, \mathrm{TR}, \mathrm{VF}, \mathrm{DM}$, and KYA acquired data and performed analysis and interpretation of

704 data. ENA wrote the manuscript. RAB and JBL reviewed and revised the manuscript. GG, Jl,

$705 \mathrm{JBL}$, and RAB supervised the study.

706

\section{Acknowledgements}

708 We thank Sissel Vik Berge, Karla Sputova, Edward Verwayen, Hallvard Haugen, Marianne

709 Enger, Michelle Scottn, Anna Boniecka, and Eline Milde for excellent technical support, and to

710 Dr. Kjell Petersen, the Computational Biology Unit, the University of Bergen, and the Norwegian

711 Bioinformatics platform for microarray analysis support. Additionally, we thank our UT

712 Southwestern colleagues Lianxin Hu and Qing Zhang for their input and technical advice. We

713 would also like to thank the UT Southwestern Proteomics core, Cheryl Lewis, director of the

714 Shared Tissue Management Resource Core (supported by P30 CA142543), and Angie Mobley,

715 manager of the Flow Cytometry core. The work was supported by NIH grants R01 CA192381,

716 R01 CA243577 and U54 CA210181 Project 2 to RAB, the Effie Marie Cain Scholarship in 


\section{Arner et al.}

717 Angiogenesis Research and the Gillson Longenbaugh Foundation to RAB, grants to JBL from

718 the Norwegian Cancer Society, Norwegian Research Council, Bergen Health Authority and

719 BerGenBio ASA, as well as CPRIT RP160157 (principal investigator: M. Cobb, UT

720 Southwestern, Dallas, Texas) and NCI F99 CA253718 to ENA. The funders had no role in study

721 design, data collection and analysis, decision to publish, or preparation of the manuscript.

722

723

724

725

726

727

728

729

730

731

732

733

734

735

736

737

738

739 
Arner et al.

\section{References}

1. Wang Z, Li Y, Ahmad A, Banerjee S, Azmi AS, Kong D, et al. Pancreatic cancer: understanding and overcoming chemoresistance. Nat Rev Gastroenterol Hepatol. 2011;8(1):27-33.

2. Tran DD, Corsa CA, Biswas H, Aft RL, and Longmore GD. Temporal and spatial cooperation of Snail1 and Twist1 during epithelial-mesenchymal transition predicts for human breast cancer recurrence. Mol Cancer Res. 2011;9(12):1644-57.

3. Seyfried TN, and Huysentruyt LC. On the origin of cancer metastasis. Crit Rev Oncog. 2013;18(1-2):43-73.

4. Cates JM, Byrd RH, Fohn LE, Tatsas AD, Washington MK, and Black CC. Epithelialmesenchymal transition markers in pancreatic ductal adenocarcinoma. Pancreas. 2009;38(1):e1-6.

5. Kudo-Saito C, Shirako H, Takeuchi T, and Kawakami Y. Cancer metastasis is accelerated through immunosuppression during Snail-induced EMT of cancer cells. Cancer Cell. 2009;15(3):195-206.

6. Puls TJ, Tan X, Whittington CF, and Voytik-Harbin SL. 3D collagen fibrillar microstructure guides pancreatic cancer cell phenotype and serves as a critical design parameter for phenotypic models of EMT. PLoS One. 2017;12(11):e0188870.

7. Larue L, and Bellacosa A. Epithelial-mesenchymal transition in development and cancer: role of phosphatidylinositol 3' kinase/AKT pathways. Oncogene. 2005;24(50):7443-54.

8. Wasif N, Ko CY, Farrell J, Wainberg Z, Hines OJ, Reber H, et al. Impact of tumor grade on prognosis in pancreatic cancer: should we include grade in AJCC staging? Ann Surg Oncol. 2010;17(9):2312-20.

9. Kirane A, Ludwig KF, Sorrelle N, Haaland G, Sandal T, Ranaweera R, et al. Warfarin Blocks Gas6-Mediated Axl Activation Required for Pancreatic Cancer Epithelial Plasticity and Metastasis. Cancer Res. 2015;75(18):3699-705.

10. Koorstra JB, Karikari CA, Feldmann G, Bisht S, Rojas PL, Offerhaus GJ, et al. The Axl receptor tyrosine kinase confers an adverse prognostic influence in pancreatic cancer and represents a new therapeutic target. Cancer Biol Ther. 2009;8(7):618-26.

11. Gjerdrum C, Tiron C, Hoiby T, Stefansson I, Haugen H, Sandal T, et al. Axl is an essential epithelial-to-mesenchymal transition-induced regulator of breast cancer metastasis and patient survival. Proc Natl Acad Sci U S A. 2010;107(3):1124-9.

12. Ludwig KF, Du W, Sorrelle NB, Wnuk-Lipinska K, Topalovski M, Toombs JE, et al. SmallMolecule Inhibition of AxI Targets Tumor Immune Suppression and Enhances Chemotherapy in Pancreatic Cancer. Cancer Res. 2018;78(1):246-55.

13. Du W, and Brekken RA. Does Axl have potential as a therapeutic target in pancreatic cancer? Expert Opin Ther Targets. 2018;22(11):955-66.

14. Cruz VH, Arner EN, Du W, Bremauntz AE, and Brekken RA. Axl-mediated activation of TBK1 drives epithelial plasticity in pancreatic cancer. JCI Insight. 2019;5. 
Arner et al.

780 15. Ou YH, Torres M, Ram R, Formstecher E, Roland C, Cheng T, et al. TBK1 directly engages Akt/PKB survival signaling to support oncogenic transformation. Mol Cell. 2011;41(4):458-70.

16. Bellacosa A, Testa JR, Staal SP, and Tsichlis PN. A retroviral oncogene, akt, encoding a serine-threonine kinase containing an SH2-like region. Science. 1991;254(5029):274-7.

17. Grille SJ, Bellacosa A, Upson J, Klein-Szanto AJ, van Roy F, Lee-Kwon W, et al. The protein kinase Akt induces epithelial mesenchymal transition and promotes enhanced motility and invasiveness of squamous cell carcinoma lines. Cancer Res. 2003;63(9):2172-8.

18. Xu W, Yang Z, and Lu N. A new role for the PI3K/Akt signaling pathway in the epithelialmesenchymal transition. Cell Adh Migr. 2015;9(4):317-24.

19. Woodgett JR. Recent advances in the protein kinase B signaling pathway. Curr Opin Cell Biol. 2005;17(2):150-7.

20. Irie HY, Pearline RV, Grueneberg D, Hsia M, Ravichandran P, Kothari N, et al. Distinct roles of Akt1 and Akt2 in regulating cell migration and epithelial-mesenchymal transition. J Cell Biol. 2005;171(6):1023-34.

21. Sanidas I, Polytarchou C, Hatziapostolou M, Ezell SA, Kottakis F, Hu L, et al. Phosphoproteomics screen reveals akt isoform-specific signals linking RNA processing to lung cancer. Mol Cell. 2014;53(4):577-90.

22. Chin YR, Yoshida T, Marusyk A, Beck AH, Polyak K, and Toker A. Targeting Akt3 signaling in triple-negative breast cancer. Cancer Res. 2014;74(3):964-73.

23. Lin FM, Yost SE, Wen W, Frankel PH, Schmolze D, Chu PG, et al. Differential gene expression and AKT targeting in triple negative breast cancer. Oncotarget. 2019;10(43):4356-68.

24. Turner KM, Sun Y, Ji P, Granberg KJ, Bernard B, Hu L, et al. Genomically amplified Akt3 activates DNA repair pathway and promotes glioma progression. Proc Natl Acad Sci U S A. 2015;112(11):3421-6.

25. Toulany M, Maier J, lida M, Rebholz S, Holler M, Grottke A, et al. Akt1 and Akt3 but not Akt2 through interaction with DNA-PKcs stimulate proliferation and post-irradiation cell survival of K-RAS-mutated cancer cells. Cell Death Discov. 2017;3:17072.

26. Lee HJ, Jeng YM, Chen YL, Chung L, and Yuan RH. Gas6/Axl pathway promotes tumor invasion through the transcriptional activation of Slug in hepatocellular carcinoma. Carcinogenesis. 2014;35(4):769-75.

27. Tang Z, Li C, Kang B, Gao G, Li C, and Zhang Z. GEPIA: a web server for cancer and normal gene expression profiling and interactive analyses. Nucleic Acids Res. 2017;45(W1):W98W102.

28. Holland SJ, Pan A, Franci C, Hu Y, Chang B, Li W, et al. R428, a selective small molecule inhibitor of Axl kinase, blocks tumor spread and prolongs survival in models of metastatic breast cancer. Cancer Res. 2010;70(4):1544-54.

29. Santi SA, and Lee $\mathrm{H}$. The Akt isoforms are present at distinct subcellular locations. Am J Physiol Cell Physiol. 2010;298(3):C580-91. al. AXL Is a Driver of Stemness in Normal Mammary Gland and Breast Cancer. iScience. 2020;23(11):101649. 
Arner et al.

823 31. Wente SR, and Rout MP. The nuclear pore complex and nuclear transport. Cold Spring Harb Perspect Biol. 2010;2(10):a000562.

32. Kosugi S, Hasebe M, Tomita M, and Yanagawa H. Systematic identification of cell cycledependent yeast nucleocytoplasmic shuttling proteins by prediction of composite motifs. Proc Natl Acad Sci U S A. 2009;106(25):10171-6.

33. Ansieau S, Collin G, and Hill L. EMT or EMT-Promoting Transcription Factors, Where to Focus the Light? Front Oncol. 2014;4:353.

34. Diaz VM, Vinas-Castells R, and Garcia de Herreros A. Regulation of the protein stability of EMT transcription factors. Cell Adh Migr. 2014;8(4):418-28.

35. Wang SP, Wang WL, Chang YL, Wu CT, Chao YC, Kao SH, et al. p53 controls cancer cell invasion by inducing the MDM2-mediated degradation of Slug. Nat Cell Biol. 2009;11(6):694-704.

36. Xiao G, Li Y, Wang M, Li X, Qin S, Sun X, et al. FBXW7 suppresses epithelial-mesenchymal transition and chemo-resistance of non-small-cell lung cancer cells by targeting snai1 for ubiquitin-dependent degradation. Cell Prolif. 2018;51(5):e12473.

37. Cuevas IC, Sahoo SS, Kumar A, Zhang H, Westcott J, Aguilar M, et al. Fbxw7 is a driver of uterine carcinosarcoma by promoting epithelial-mesenchymal transition. Proc Natl Acad Sci U S A. 2019;116(51):25880-90.

38. Yang $H$, Lu X, Liu Z, Chen L, Xu Y, Wang Y, et al. FBXW7 suppresses epithelialmesenchymal transition, stemness and metastatic potential of cholangiocarcinoma cells. Oncotarget. 2015;6(8):6310-25.

39. Hudis C, Swanton C, Janjigian YY, Lee R, Sutherland S, Lehman R, et al. A phase 1 study evaluating the combination of an allosteric AKT inhibitor (MK-2206) and trastuzumab in patients with HER2-positive solid tumors. Breast Cancer Res. 2013;15(6):R110.

40. Yap TA, Yan L, Patnaik A, Tunariu N, Biondo A, Fearen I, et al. Interrogating two schedules of the AKT inhibitor MK-2206 in patients with advanced solid tumors incorporating novel pharmacodynamic and functional imaging biomarkers. Clin Cancer Res. 2014;20(22):5672-85.

41. Ma BB, Goh BC, Lim WT, Hui EP, Tan EH, Lopes Gde L, et al. Multicenter phase II study of the AKT inhibitor MK-2206 in recurrent or metastatic nasopharyngeal carcinoma from patients in the mayo phase II consortium and the cancer therapeutics research group (MC1079). Invest New Drugs. 2015;33(4):985-91.

42. Jansen VM, Mayer IA, and Arteaga CL. Is There a Future for AKT Inhibitors in the Treatment of Cancer? Clin Cancer Res. 2016;22(11):2599-601. and characterization of pleckstrin-homology-domain-dependent and isoenzyme-specific Akt inhibitors. Biochem J. 2005;385(Pt 2):399-408.

44. Lindsley CW, Zhao Z, Leister WH, Robinson RG, Barnett SF, Defeo-Jones D, et al. Allosteric Akt (PKB) inhibitors: discovery and SAR of isozyme selective inhibitors. Bioorg Med Chem Lett. 2005;15(3):761-4.

45. Wu WI, Voegtli WC, Sturgis HL, Dizon FP, Vigers GP, and Brandhuber BJ. Crystal structure of human AKT1 with an allosteric inhibitor reveals a new mode of kinase inhibition. PLoS One. 2010;5(9):e12913. 
Arner et al.

46. Cao D, Maitra A, Saavedra JA, Klimstra DS, Adsay NV, and Hruban RH. Expression of novel markers of pancreatic ductal adenocarcinoma in pancreatic nonductal neoplasms: additional evidence of different genetic pathways. Mod Pathol. 2005;18(6):752-61.

47. Anwar T, Rufail ML, Djomehri SI, Gonzalez ME, Lazo de la Vega L, Tomlins SA, et al. Nextgeneration sequencing identifies recurrent copy number variations in invasive breast carcinomas from Ghana. Mod Pathol. 2020.

48. Meric-Bernstam F, Frampton GM, Ferrer-Lozano J, Yelensky R, Perez-Fidalgo JA, Wang Y, et al. Concordance of genomic alterations between primary and recurrent breast cancer. Mol Cancer Ther. 2014;13(5):1382-9.

49. Bonin S, Pracella D, Barbazza R, Dotti I, Boffo S, and Stanta G. PI3K/AKT Signaling in Breast Cancer Molecular Subtyping and Lymph Node Involvement. Dis Markers. 2019;2019:7832376.

50. Konishi H, Kuroda S, Tanaka M, Matsuzaki H, Ono Y, Kameyama K, et al. Molecular cloning and characterization of a new member of the RAC protein kinase family: association of the pleckstrin homology domain of three types of RAC protein kinase with protein kinase $\mathrm{C}$ subspecies and beta gamma subunits of $\mathrm{G}$ proteins. Biochem Biophys Res Commun. 1995;216(2):526-34.

51. Santi SA, and Lee H. Ablation of Akt2 induces autophagy through cell cycle arrest, the downregulation of p70S6K, and the deregulation of mitochondria in MDA-MB231 cells. PLoS One. 2011;6(1):e14614.

52. Stottrup C, Tsang T, and Chin YR. Upregulation of AKT3 Confers Resistance to the AKT Inhibitor MK2206 in Breast Cancer. Mol Cancer Ther. 2016;15(8):1964-74.

53. Li Y, Cai B, Shen L, Dong Y, Lu Q, Sun S, et al. MiRNA-29b suppresses tumor growth through simultaneously inhibiting angiogenesis and tumorigenesis by targeting Akt3. Cancer Lett. 2017;397:111-9.

54. Grottke A, Ewald F, Lange T, Norz D, Herzberger C, Bach J, et al. Downregulation of AKT3 Increases Migration and Metastasis in Triple Negative Breast Cancer Cells by Upregulating S100A4. PLoS One. 2016;11(1):e0146370.

55. Chung S, Yao J, Suyama K, Bajaj S, Qian X, Loudig OD, et al. N-cadherin regulates mammary tumor cell migration through Akt3 suppression. Oncogene. 2013;32(4):42230.

56. Mure H, Matsuzaki K, Kitazato KT, Mizobuchi Y, Kuwayama K, Kageji T, et al. Akt2 and Akt3 play a pivotal role in malignant gliomas. Neuro Oncol. 2010;12(3):221-32.

57. Nakatani K, Sakaue H, Thompson DA, Weigel RJ, and Roth RA. Identification of a human Akt3 (protein kinase $B$ gamma) which contains the regulatory serine phosphorylation site. Biochem Biophys Res Commun. 1999;257(3):906-10.

58. Nakatani K, Thompson DA, Barthel A, Sakaue H, Liu W, Weigel RJ, et al. Up-regulation of Akt3 in estrogen receptor-deficient breast cancers and androgen-independent prostate cancer lines. J Biol Chem. 1999;274(31):21528-32.

59. Stahl JM, Sharma A, Cheung M, Zimmerman M, Cheng JQ, Bosenberg MW, et al. Deregulated Akt3 activity promotes development of malignant melanoma. Cancer Res. 2004;64(19):7002-10. 
Arner et al.

908

909

910

911

912

913

914

915

916

917

918

919

920

921

922

923

924

925

926

927

928

929

930

931

932

933

934

935

936

937

938

939

940

941

942

943

944

945

946

947

948

949

950

60. Suyama K, Yao J, Liang H, Benard O, Loudig OD, Amgalan D, et al. An Akt3 Splice Variant Lacking the Serine 472 Phosphorylation Site Promotes Apoptosis and Suppresses Mammary Tumorigenesis. Cancer Res. 2018;78(1):103-14.

61. Ouchida AT, Kacal M, Zheng A, Ambroise G, Zhang B, Norberg E, et al. USP10 regulates the stability of the EMT-transcription factor Slug/SNAI2. Biochem Biophys Res Commun. 2018;502(4):429-34.

62. $\mathrm{Yu} \mathrm{Q}, \mathrm{Zhou} \mathrm{BP}$, and $\mathrm{Wu} \mathrm{Y}$. The regulation of snail: on the ubiquitin edge. Cancer Cell Microenviron. 2017;4(2).

63. Dang TT, Esparza MA, Maine EA, Westcott JM, and Pearson GW. DeltaNp63alpha Promotes Breast Cancer Cell Motility through the Selective Activation of Components of the Epithelial-to-Mesenchymal Transition Program. Cancer Res. 2015;75(18):3925-35.

64. Xiao J, Li W, Zheng X, Qi L, Wang H, Zhang C, et al. Targeting 7-Dehydrocholesterol Reductase Integrates Cholesterol Metabolism and IRF3 Activation to Eliminate Infection. Immunity. 2020;52(1):109-22 e6.

65. Gu S, Dai H, Zhao X, Gui C, and Gui J. AKT3 deficiency in M2 macrophages impairs cutaneous wound healing by disrupting tissue remodeling. Aging (Albany NY). 2020;12.

66. Swift S, Lorens J, Achacoso P, and Nolan GP. Rapid production of retroviruses for efficient gene delivery to mammalian cells using 293T cell-based systems. Curr Protoc Immunol. 2001; Chapter 10:Unit 10 7C.

67. Labarge MA, Garbe JC, and Stampfer MR. Processing of human reduction mammoplasty and mastectomy tissues for cell culture. J Vis Exp. 2013(71).

68. Garbe JC, Pepin F, Pelissier FA, Sputova K, Fridriksdottir AJ, Guo DE, et al. Accumulation of multipotent progenitors with a basal differentiation bias during aging of human mammary epithelia. Cancer Res. 2012;72(14):3687-701.

69. Mani SA, Guo W, Liao MJ, Eaton EN, Ayyanan A, Zhou AY, et al. The epithelialmesenchymal transition generates cells with properties of stem cells. Cell. 2008;133(4):704-15.

70. Vuoriluoto K, Haugen H, Kiviluoto S, Mpindi JP, Nevo J, Gjerdrum C, et al. Vimentin regulates EMT induction by Slug and oncogenic $\mathrm{H}$-Ras and migration by governing $\mathrm{Axl}$ expression in breast cancer. Oncogene. 2011;30(12):1436-48.

71. Westcott JM, Prechtl AM, Maine EA, Dang TT, Esparza MA, Sun H, et al. An epigenetically distinct breast cancer cell subpopulation promotes collective invasion. $J$ Clin Invest. 2015;125(5):1927-43.

72. Dontu G, Abdallah WM, Foley JM, Jackson KW, Clarke MF, Kawamura MJ, et al. In vitro propagation and transcriptional profiling of human mammary stem/progenitor cells. Genes Dev. 2003;17(10):1253-70.

73. Kim D, Paggi JM, Park C, Bennett C, and Salzberg SL. Graph-based genome alignment and genotyping with HISAT2 and HISAT-genotype. Nat Biotechnol. 2019;37(8):907-15.

74. Liao Y, Smyth GK, and Shi W. featureCounts: an efficient general purpose program for assigning sequence reads to genomic features. Bioinformatics. 2014;30(7):923-30.

75. McCarthy DJ, Chen Y, and Smyth GK. Differential expression analysis of multifactor RNASeq experiments with respect to biological variation. Nucleic Acids Res. 2012;40(10):4288-97. 
Arner et al.

951 76. Robinson MD, McCarthy DJ, and Smyth GK. edgeR: a Bioconductor package for

952

953

954

955

956

957

958

959

960

961

962

963

964

965

966

967

968

969

970

971

972

973

974

975

976

977

978

979

980

981

982

983

984

985

986

987

988

989

990

991

992 differential expression analysis of digital gene expression data. Bioinformatics. 2010;26(1):139-40.

77. Curtis C, Shah SP, Chin SF, Turashvili G, Rueda OM, Dunning MJ, et al. The genomic and transcriptomic architecture of 2,000 breast tumours reveals novel subgroups. Nature. 2012;486(7403):346-52.

78. Parker JS, Mullins M, Cheang MC, Leung S, Voduc D, Vickery T, et al. Supervised risk predictor of breast cancer based on intrinsic subtypes. J Clin Oncol. 2009;27(8):1160-7.

79. Bae SS, Cho H, Mu J, and Birnbaum MJ. Isoform-specific regulation of insulin-dependent glucose uptake by Akt/protein kinase B. J Biol Chem. 2003;278(49):49530-6.

80. Gupta PB, Onder TT, Jiang G, Tao K, Kuperwasser C, Weinberg RA, et al. Identification of selective inhibitors of cancer stem cells by high-throughput screening. Cell. 2009;138(4):645-59.

81. Huang H, Zhang Y, Gallegos V, Sorrelle N, Zaid MM, Toombs J, et al. Targeting TGFbetaR2-mutant tumors exposes vulnerabilities to stromal TGFbeta blockade in pancreatic cancer. EMBO Mol Med. 2019;11(11):e10515.

82. Lieber M, Mazzetta J, Nelson-Rees W, Kaplan M, and Todaro G. Establishment of a continuous tumor-cell line (panc-1) from a human carcinoma of the exocrine pancreas. Int J Cancer. 1975;15(5):741-7.

83. Brinkley BR, Beall PT, Wible LJ, Mace ML, Turner DS, and Cailleau RM. Variations in cell form and cytoskeleton in human breast carcinoma cells in vitro. Cancer Res. 1980;40(9):3118-29.

84. Cailleau R, Olive M, and Cruciger QV. Long-term human breast carcinoma cell lines of metastatic origin: preliminary characterization. In Vitro. 1978;14(11):911-5.

85. Siciliano MJ, Barker PE, and Cailleau R. Mutually exclusive genetic signatures of human breast tumor cell lines with a common chromosomal marker. Cancer Res. 1979;39(3):919-22.

86. Soule HD, Maloney TM, Wolman SR, Peterson WD, Jr., Brenz R, McGrath CM, et al. Isolation and characterization of a spontaneously immortalized human breast epithelial cell line, MCF-10. Cancer Res. 1990;50(18):6075-86.

87. Tait L, Soule HD, and Russo J. Ultrastructural and immunocytochemical characterization of an immortalized human breast epithelial cell line, MCF-10. Cancer Res. 1990;50(18):6087-94.

88. Tripathi MK, and Chaudhuri G. Down-regulation of UCRP and UBE2L6 in BRCA2 knockeddown human breast cells. Biochem Biophys Res Commun. 2005;328(1):43-8.

89. Tripathi MK, Misra S, Khedkar SV, Hamilton N, Irvin-Wilson C, Sharan C, et al. Regulation of BRCA2 gene expression by the SLUG repressor protein in human breast cells. J Biol Chem. 2005;280(17):17163-71.

90. Elenbaas B, Spirio L, Koerner F, Fleming MD, Zimonjic DB, Donaher JL, et al. Human breast cancer cells generated by oncogenic transformation of primary mammary epithelial cells. Genes Dev. 2001;15(1):50-65. 


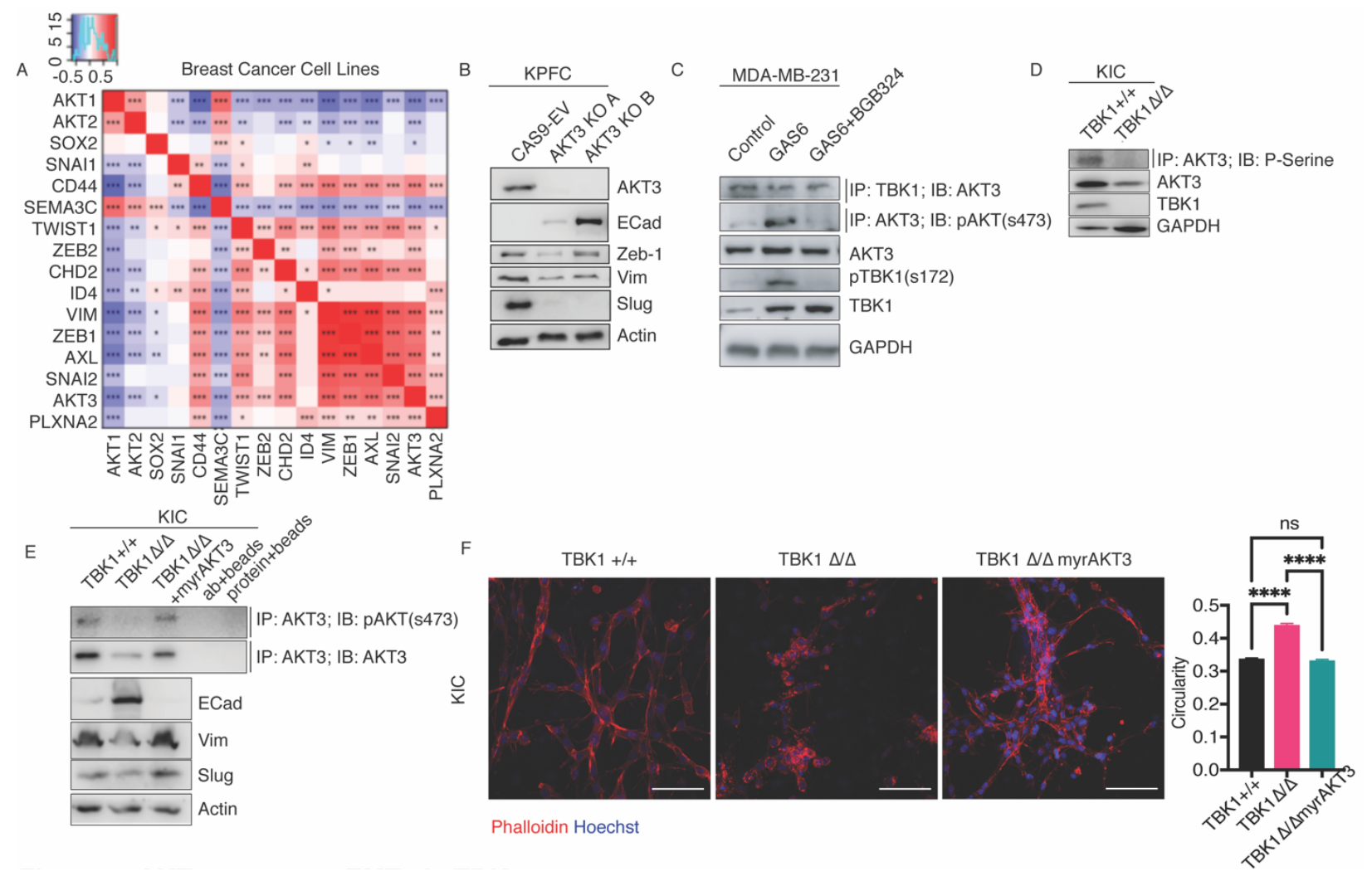

Figure 1. TBK1 activates AKT3 to promote EMT

A) Correlation of AKT1, AKT2, and AKT3 mRNA with EMT and stem cell associated genes in breast cancer cell lines. Positive correlation values are demarcated as red and negative correlation values are shown as blue $\left({ }^{*} p<0.045,{ }^{* *} p<0.009,{ }^{* * *} p<2 \times 10^{-5}\right.$; Spearman's correlation test). B) Western blot for the indicated target of KPFC PDA control Cas9- empty vector (CAS9-EV) or AKT3 CRISPR-mediated deletion (KO A, KO B). C) MDA-MB-231 cells were stimulated with DMSO, GAS6 $(200 \mathrm{ng} / \mathrm{ml})+/-2 \mu \mathrm{M}$ BGB324. Immunoprecipitation of AKT3 was probed for pAKT(s473) and immunoprecipitation of TBK1 was probed for AKT3. Total lysates were probed for AKT3, pTBK1 (s172), TBK1 and GAPDH (loading control). D) Immunoprecipitation of AKT3 in TBK1 WT and mutant $\left(T B K 1^{\Delta / \Delta}\right) K I C$ PDA cells probed for total phospho-serine. Total lysates were probed for AKT3, TBK1 and Actin (loading control). E) Immunoprecipitation of AKT3 in $T B K 1^{+/+}, T B K 1^{\Delta / \Delta}$, and $T B K 1^{\Delta / \Delta}$ KIC PDA cells transduced with myrAKT3 (TBK1 $1^{\Delta / \Delta}$ - myrAKT3). AKT3 immunoprecipitation was probed for pAKT (s473) and total AKT3. Cell lysates were probed for E Cadherin, vimentin, slug and actin (loading control). Immunoprecipitation controls without protein or antibody are shown. F) $T B K 1^{+/+}, T B K 1^{\Delta / \Delta}$, and $T B K 1^{\Delta / \Delta}$-myrAKT3 cells were plated in collagen/matrigel and stained for Phalloidin (red) and Hoechst (blue). Z-stack $(1 \mu \mathrm{m})$ images were taken by confocal microscopy at 20X magnification. Scale bar, $100 \mu \mathrm{m}$. Circularity of cells was calculated using ImageJ. $\mathrm{n}>500$ cells/condition. All statistics were done using one-way ANOVA; ${ }^{*} p<0.05$, ${ }^{* *} p<0.01,{ }^{* * *} p<0.001$, ${ }^{* * * *} p<0.0001$. All representative results shown were reproduced in at least three independent experiments. representative results shown were reproduced in at least three independent experiments. 


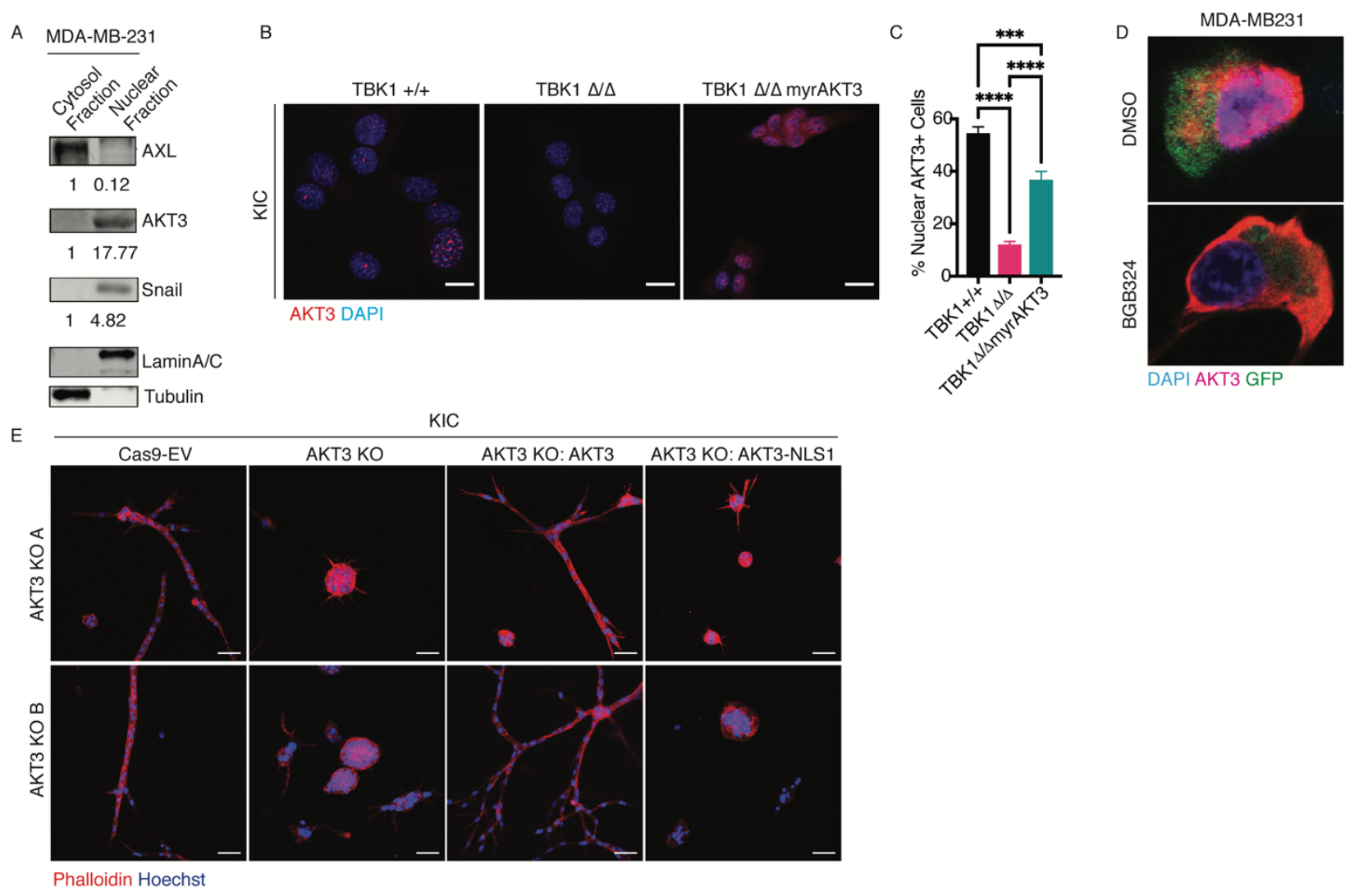

Figure 2. AXL-TBK1 is required for AKT3 nuclear localization

A) Western blot analysis of AXL, AKT3, snail, lamin A/C and tubulin in MDA-MB-231 nuclear and cytoplasmic fractions. Protein expression was quantified and normalized to protein in the cytosol. B-C) Immunofluorescence of AKT3 (red) and DAPI (blue) in $T B K 1^{+/+}, T B K 1^{\Delta / \Delta}$, and $T B K 1^{\Delta / \Delta}$-myrAKT3 KIC PDA cells. Percent of cells with nuclear AKT3 is graphed in (C). Scale bar, $20 \mu \mathrm{m}$. D) Immunofluorescence of MDA-MB-231/GFP cells treated with DMSO or BGB324. Cells are stained with AKT3 (red) and nuclei are stained with DAPI (blue). E) KPFC CAS9-EV, AKT3 KO, AKT3 KO cells transduced with AKT3, and AKT3 KO cells transduced with AKT3NLS1 mutant plated in collagen/matrigel and stained with Phalloidin (red) and Hoechst (blue). Zstack $(1 \mu \mathrm{m})$ images were taken by confocal microscopy at 20X magnification. Cells were imaged at 20X using confocal microscopy. Scale bar, $50 \mu \mathrm{m}$. Two different AKT3 KO CRISPR clones are displayed. All representative results shown were reproduced in at least three independent experiments. All statistics were done use one-way ANOVA: ${ }^{* *}, p<0.001 ;{ }^{* * *}, p$ $<0.0001$. 
A

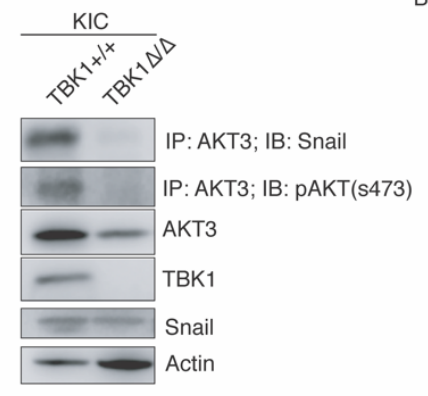

B

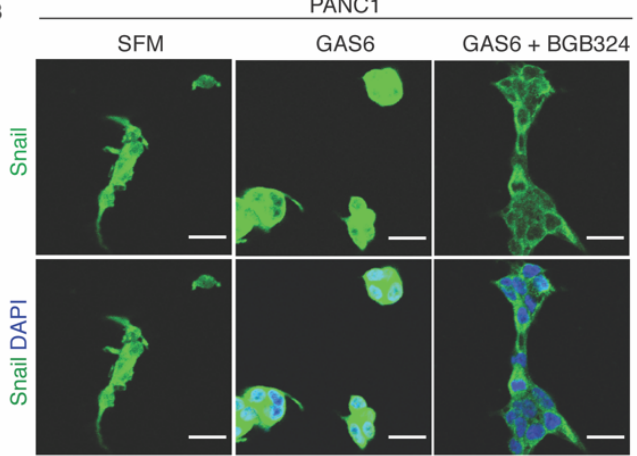

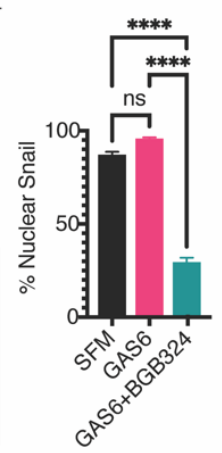
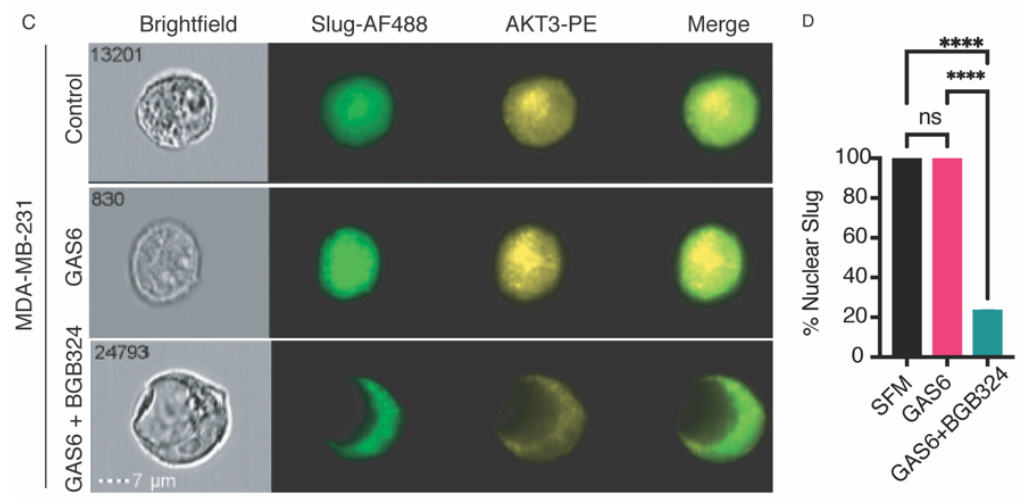

Figure 3. Snail/Slug is a TBK1-dependent substrate of AKT3

A) Immunoprecipitation of AKT3 in primary TBK1 WT and mutant $\left(T B K 1^{\Delta / \Delta}\right) K I C$ cells were probed for snail and pAKT (s473). Lysates were probed for total snail, AKT3, TBK1, and Actin. B) Immunofluorescence of snail (green) and DAPI (blue) in PANC1 cells treated with serum free media (SFM), $200 \mathrm{ng} / \mathrm{mL}$ GAS6 +/- $2 \mu \mathrm{M}$ BGB324 for $12 \mathrm{hrs}$. Cells were imaged at 20X using confocal microscopy (scale bar, $20 \mu \mathrm{m}$ ) and nuclear snail was quantified, $\mathrm{n}>200$ cells. C) Representative images from Imaging Flow cytometry (Amnis Imagestream ${ }^{\circledR}$ ) of Slug-AF488 (green) and AKT3-PE (yellow) inMDA-MB-231 cells treated with $200 \mathrm{ng} / \mathrm{mL}$ GAS6 +/- $2 \mu \mathrm{M}$ BGB324 for $6 \mathrm{hrs}$. Scale bar, $7 \mu \mathrm{m}$. D) Nuclear localization of slug was quantified in each condition from (C); Control, $n=549$, GAS6, $n=6781$, GAS6 + BGB324, $n=7730$. Slug and AKT3 co-localization was quantified. All representative results shown were reproduced in at least three independent experiments. All statistics were done use one-way ANOVA: ${ }^{* * *}, p<0.0001$. 
A

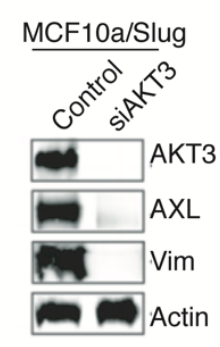

B
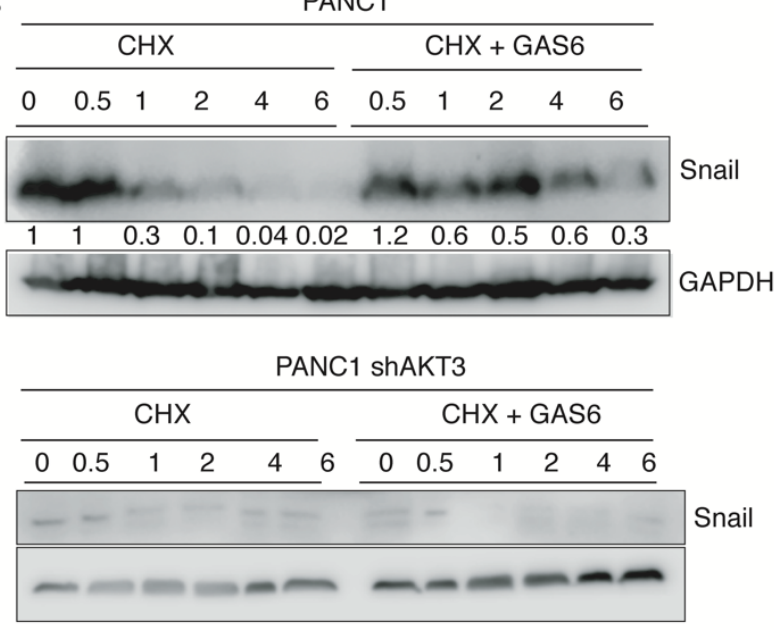

C

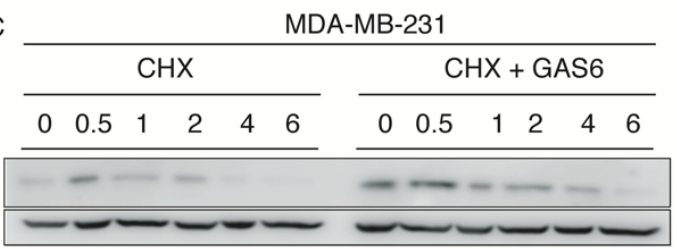

MDA-MB-231 shAKT3

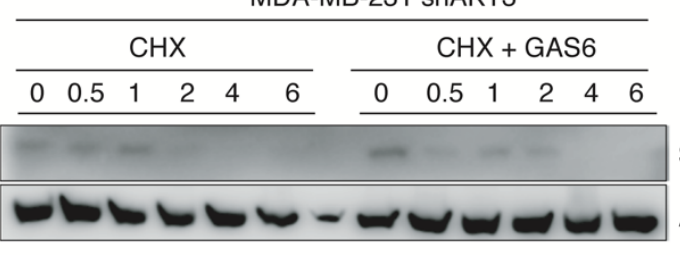

D

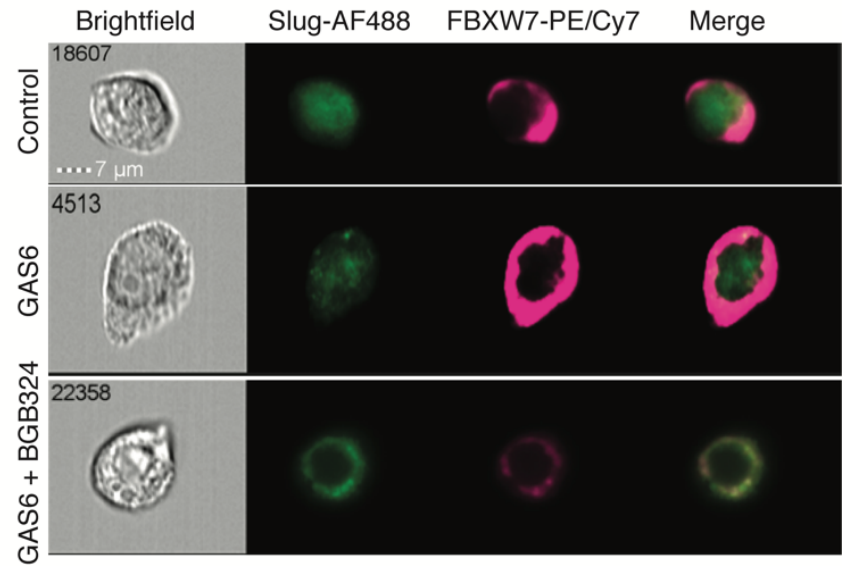

Figure 4. AXL activity stabilizes snail/slug via AKT3

A) MCF10a/Slug cells transfected with siAKT3 or control. Lysates were probed for AKT3, AXL, vimentin, and actin. B,C) PANC1 (B) and MDA-MB-231 (C) cells transduced with shAKT3 were treated with cycloheximide $(\mathrm{CHX}, 0.5 \mu \mathrm{g} / \mathrm{mL})$ or $\mathrm{CHX}+\mathrm{GAS} 6(200 \mathrm{ng} / \mathrm{mL})$ and harvested at 30 min, 1, 2, 4, and $6 \mathrm{hrs}$ of treatment. Lysates were probed for snail/slug and GAPDH/actin. D) Representative images from imaging flow cytometry (Amnis Imagestream $®$ ) of Slug-AF488 (green) and FBXW7-Pe/Cy7 (pink) in MDA-MB-231 cells untreated or treated with $200 \mathrm{ng} / \mathrm{mL}$ GAS6 +/- $2 \mu \mathrm{M}$ BGB324 for $6 \mathrm{hrs}$. Scale bar, $7 \mu \mathrm{m}$. All representative results shown were reproduced in at least three independent experiments. 

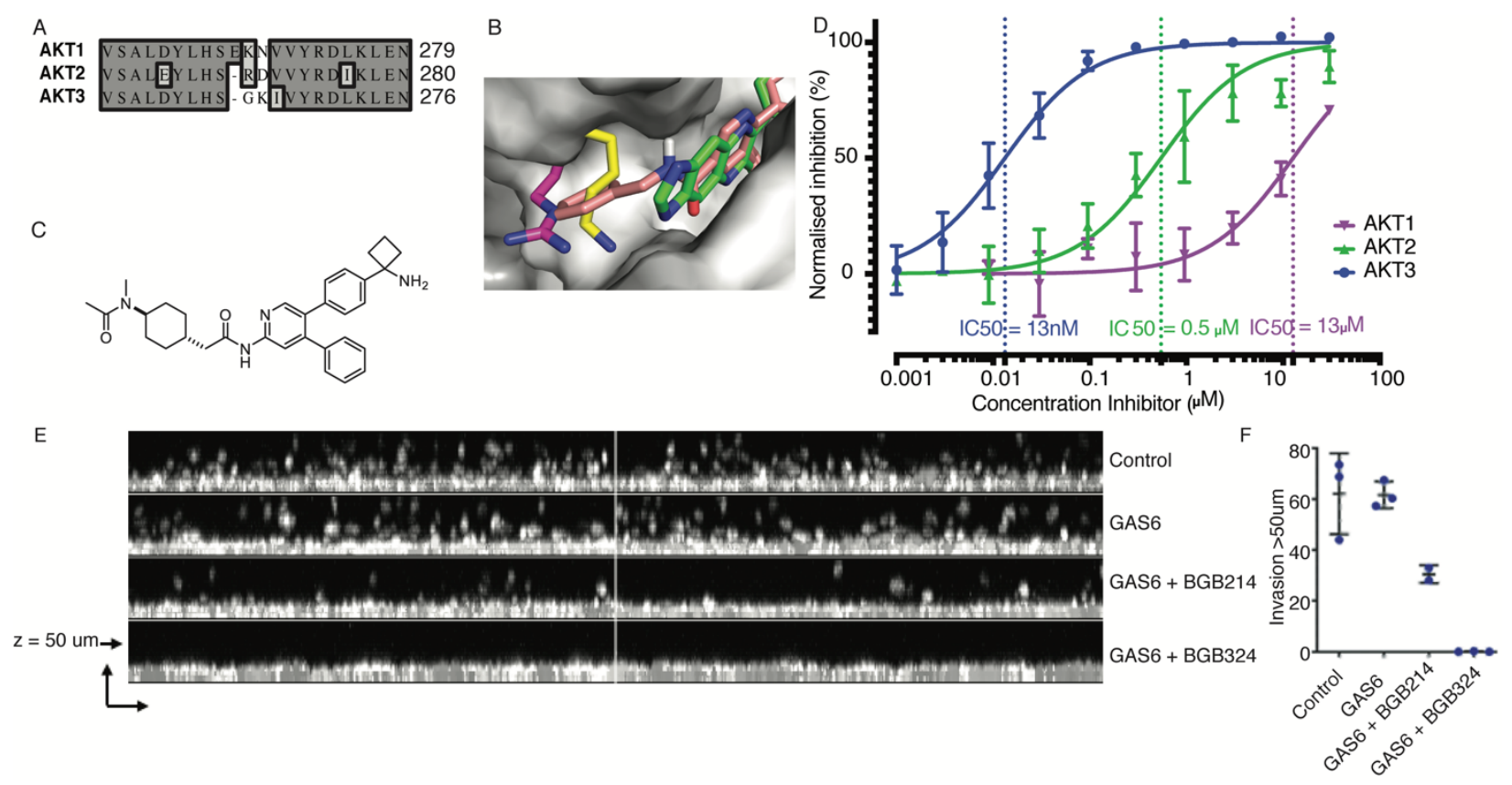

Figure 5. Efficacy of selective targeting of AKT3 with a novel allosteric small molecule inhibitor.

A) Exploitable differences in sequence between AKT1, AKT2 and AKT3 around the allosteric site include a deletion in AKT2 and AKT3 compared to AKT1, implying that the backbone may follow a different path in these proteins. B) Surface view of the front of the allosteric binding site of AKT3, including bound allosteric inhibitor AKT VIII (green). Homology model of AKT3 based on crystal structures of AKT1 bound to AKT VIII (PDB 3096) and AKT2 kinase domain (PDB106k). Side chains from the AKT1 crystal structure (Lys268, yellow) and the AKT2 crystal structure (Arg269, magenta) are superimposed, showing how they impinge on the space made available by the smaller glycine present at this location in AKT3 (Gly265). A molecule with similar structure to BGB214 (pink) docked at the allosteric site clashes with Lys268 of AKT1 (yellow). C) Structure of BGB214. D) Inhibition of AKT1, AKT2 and AKT3 enzymatic activity on GSK3a-derived Ultra U lightTM-labelled crosstide substrate $(n>3)$. E) MDA-MB-231 cells plated in collagen/matrigel and treated with GAS6 +/- $3 \mu \mathrm{M}$ BGB214 or $2 \mu \mathrm{M}$ BGB324 for $48 \mathrm{hrs}$. Zstack images were taken using confocal microscopy over $50 \mu \mathrm{m}$. F) Invasion greater than 50 $\mu \mathrm{m}$ was quantified. 


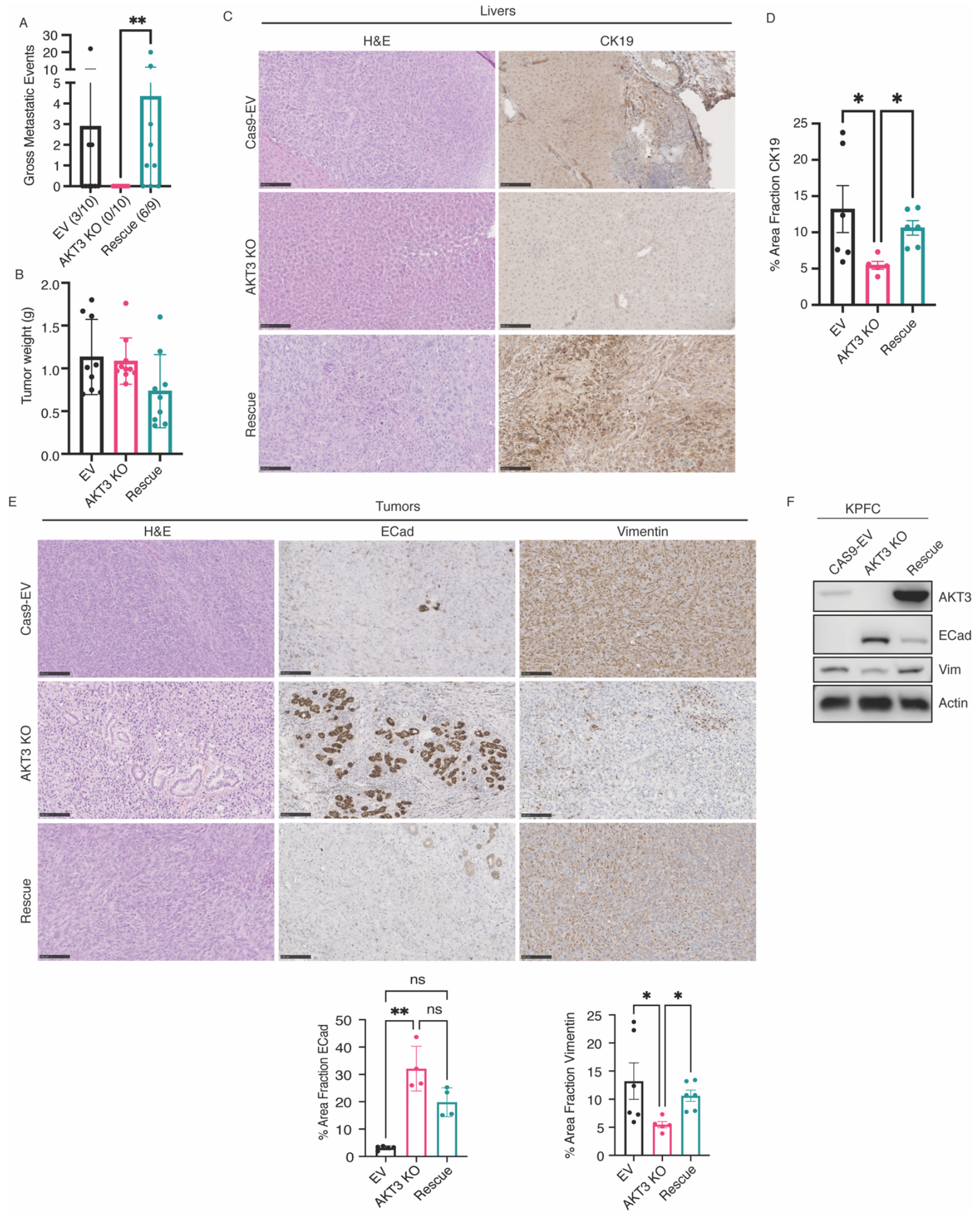

Figure 6. AKT3 expression is associated with poorly differentiated tumors and increased metastasis.

C57BL/6J mice were injected orthotopically with 250,000 KPFC PDA cells (CAS9-EV, AKT3 KO, or Rescue). A) Gross metastases (CAS9-EV: 3/9; AKT3 KO: 0/10; Rescue: 6/9) and B) 
primary tumor weight was evaluated 19 days after tumor cell injection. C-D) Representative images of H\&Es and CK19 IHC on livers. CK19 reactivity was quantified as percent of total liver area. E) Representative images of tumors stained using H\&E and IHC for E-Cadherin and vimentin. Percent area of Ecad and vimentin was quantified. F) Western blot of KPFC CAS9EV, AKT3 KO, and Rescue cells. Cells were lysed and probed for AKT3, ECad, vimentin, and actin (loading control). All statistics were done using one-way ANOVA: ${ }^{*}, p<0.05 ;{ }^{* *}, p<0.01$. 

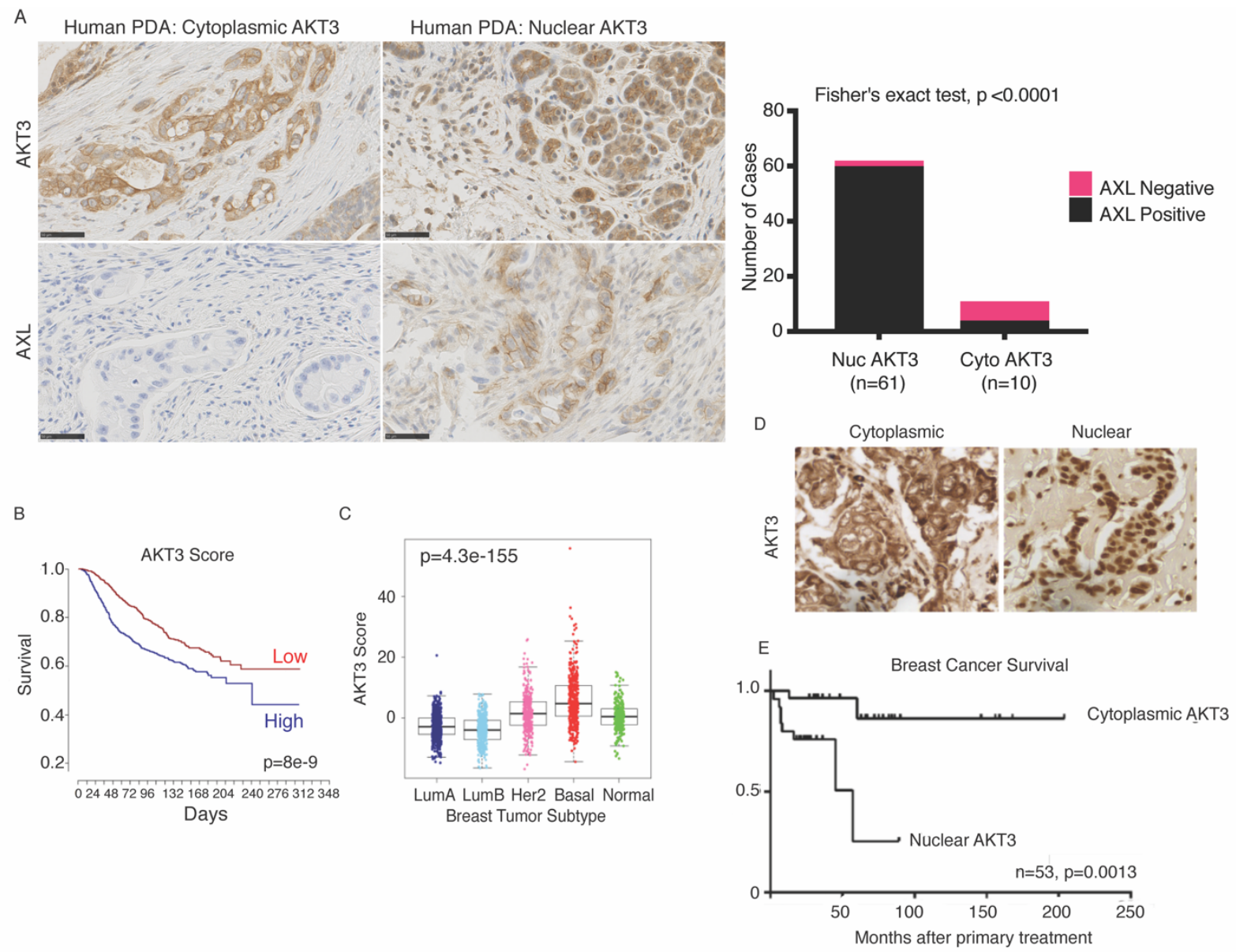

Figure 7. Nuclear AKT3 is associated with aggressive cancer and worse survival in patients.

Representative images of IHC staining for AKT3 and AXL in human PDA $(n=71)$. Fisher's exact contingency test was used to calculate if there was a correlation between nuclear AKT3 and AXL positive expression within the tumor. B) Kaplan Meier plot indicating that high AKT3 induced expression (AKT3 score) correlated with worse outcome $(p=8 e-9)$ based on the METABRIC database. C) Significant different distribution of the AKT3 score between the PAM50 subtypes ( $p=4.3 e-115$, Kruskal-Wallis test); ER negative tumors were in general enriched for the AKT3 score, particularly Basal-like tumors. D) Representative images of IHC staining for AKT3 in human breast cancer samples in (E). AKT3 localization predominantly cytoplasmic (left) or nuclear (right). E) Survival analyses of 53 breast cancer patients based on nuclear or cytoplasmic AKT3 localization ( $p=0.0013$ Log rank (Mantel-Cox) test). 


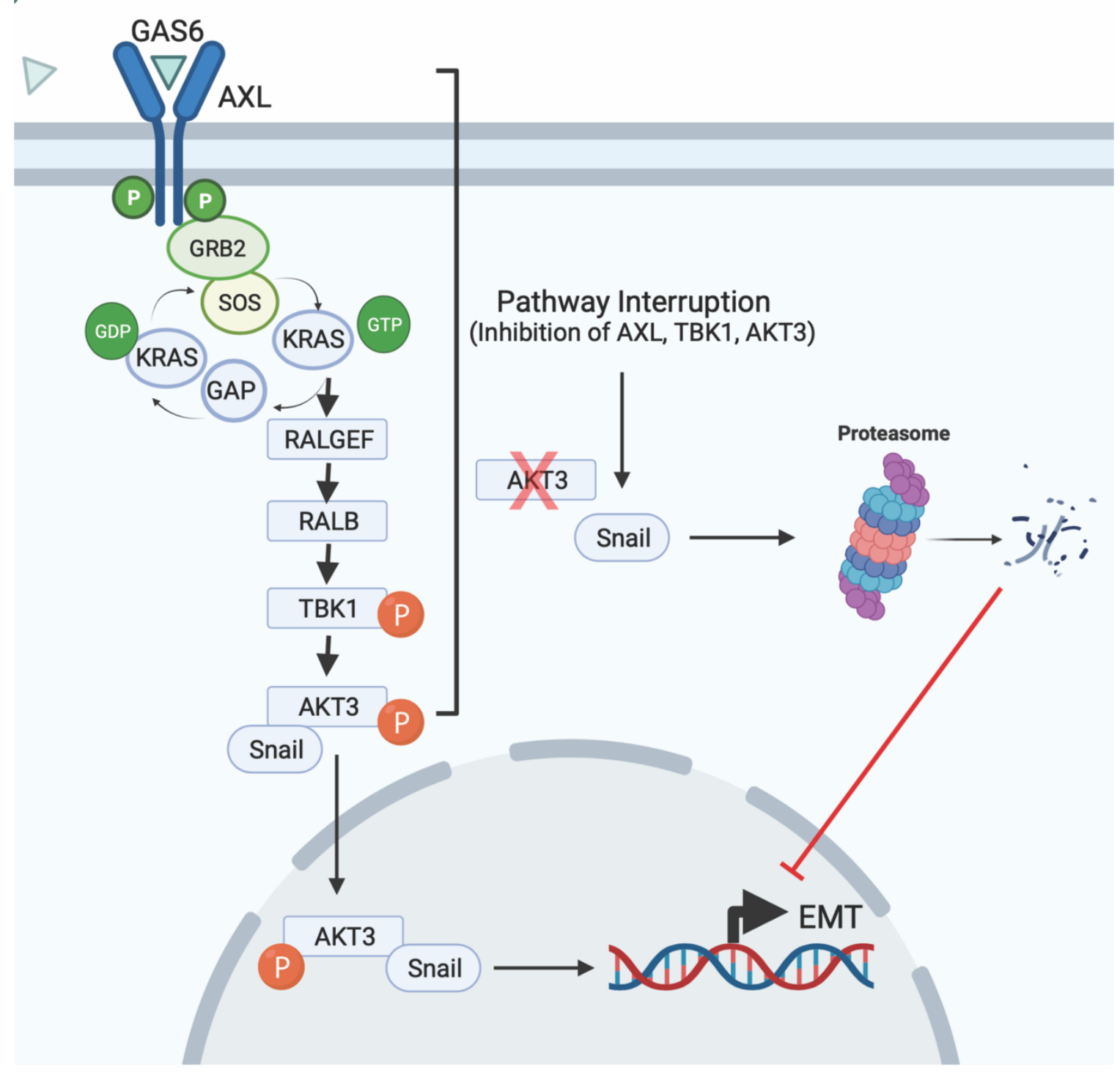

Figure 8. AXL-TBK1 driven nuclear AKT3 stabilizes slug and snail to promote EMT.

AXL activation by its ligand GAS6 leads to the stimulation of TBK1 and subsequent activation of AKT3. Activation of AKT3 drives the binding of AKT3 to slug/snail, where they are translocated into the nucleus. The binding of AKT3 to slug/snail protects the EMT-TFs from proteasomal degradation. When this pathway is interrupted and AKT3 is not activated, AKT3 can no longer bind to slug/snail thus leading to proteasomal degradation of the EMT-TFs and a decrease in EMT. 